\title{
High-fidelity prediction of crack formation in 2D and 3D pullout tests
}

\author{
Lorenzo Benedetti, Miguel Cervera, Michele Chiumenti \\ International Center for Numerical Methods in Engineering (CIMNE), \\ Technical University of Catalonia (UPC), Edificio C1, Campus Norte, \\ Jordi Girona 1-3, 08034 Barcelona, Spain \\ Corresponding author: lbenedetti@cimne.upc.edu
}

\begin{abstract}
This paper presents the 2D and 3D numerical analysis of pullout tests on steel anchorages in concrete blocks using standard and mixed finite elements. A novel (stabilized) mixed formulation in the variables of total strain $\varepsilon$ and displacements $u$ is introduced to overcome the intrinsic deficiencies of the standard displacement-based one in the context of localization of strains, such as mesh dependency. The quasi-brittle behaviour of concrete is described through an elastoplastic constitutive law with a local Rankine yielding criterion. The proposed formulation is shown to be a reliable and accurate tool, sensitive to the physical parameters of the pullout tests, but objective with respect to the adopted FE mesh. Furthermore, the mixed $\varepsilon / u$ finite element is able to capture the correct failure mechanism with relatively coarse discretizations. At the same time, the spurious behavior of the standard formulation is not alleviated by mesh-refinement.
\end{abstract}




\section{Introduction}

Concrete is widely used in the context of civil structures, and yet, it is a rather complex material. Having an inherent internal heterogeneity and reacting to environmental conditions makes the postpeak non-linear behavior and the subsequent failure very difficult to predict, if not impossible. Then, it is clear the key role of experimental tests in the context of concrete structures reliability. Within the extensive literature in the field, one can recall L-shaped panels tests by Winkler et al. [1], wedge splitting by Trunk [2], single edge notched beams by Guinea et al. [3], Gálvez et al. [4], mixed mode fracture tests by Nooru-Mohamed [5], Ballatore et al. [6] and indentation tests by Berenbaum and Brodie [7].

In this work, the pullout test is addressed, being one of the most interesting experimental techniques to evaluate the strength of concrete and the overall behavior of embedded anchorages. First accounts of the pullout test come from Abrams [8] and Slater et al. [9]. Later, Elfgren et al. [10] and Peier [11] introduced numerical modeling as a validation of the experimental procedure. While the laboratory setup can be easily reproduced, the test outcomes are strictly dependent on the choice of various parameters as the mechanical properties of the employed materials, the size of specimens and the anchorage embed depth. The influential works of Eligchausen and Sawade [12], Bažant et al. [13]. Ožbolt et al. [14], Karihaloo [15] emphasized the structural size effect on the pullout test and its influence on the energy dissipated in the cracking process. Most recent advances are related to the possibility of testing the coupling between FRP composites and concrete [16, 17]. Hereafter, the attention will be focused on the reported experiments from Dejori [18], Thenier and Hofstetter [19] for the 2D case, whereas the work by Gasser and Holzapfel [20], Areias and Belytschko [21] will be considered for the 3D case.

The methodology used to assess physical properties of specimens during a test is as critical as

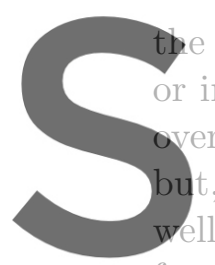
details that characte in the supporting syster multiple experiment in reality, not all con as time and cost restri
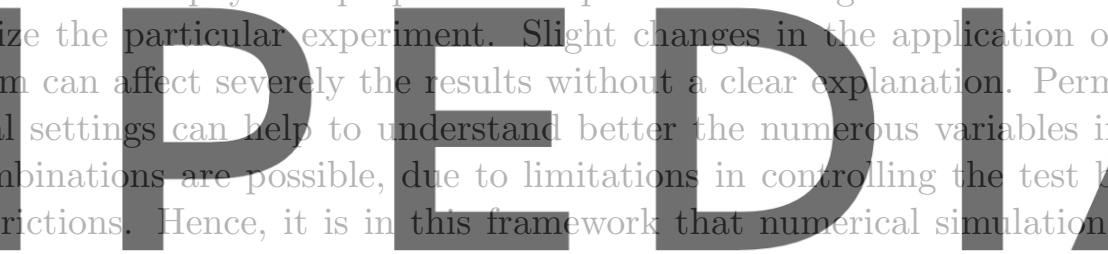

fundamental role for the prediction and the possible improvement of experiments.

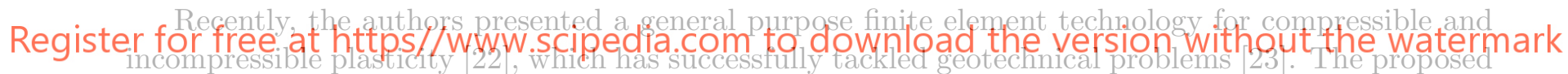

mixed strain-displacement $(\varepsilon / u)$ formulation has been applied to local constitutive models in plasticity, in the framework of the smeared crack approach [24]. In problems involving strain-localization, standard finite elements present numerous limitations, being affected by spurious mesh-biased dependence and stress locking. In such cases, the sensibility required to evaluate the change of results with respect to diverse boundary conditions can be overshadowed by the lack of precision in the inelastic range of classical displacement-based finite elements. On the contrary, the mixed $\varepsilon / u$ finite element formulation is capable to overcome these issues, predicting effectively the peak load, the failure mechanism and the localization bands. The method is also free from any mesh dependence and it does not require any additional tracking technique. This is a substantial clinching feat, as, leaving out theoretical qualms and from the factual point of view, local crack tracking procedures are very difficult to implement in 3D and global methods cannot deal with crack branching.

Taking advantage of its reliability, the proposed method is applied in this work to the pullout problem, using 2D and 3D elements with linear interpolations of both the displacement and total strain fields. In previous works $[25,26,27]$, the formulation has been used in the context of isotropic damage models. In this work, though, a plasticity model based on the Rankine failure criterion is used, similarly to those used in references [20, 28, 29, 30, 31]. Furthermore, plasticity with strain softening has been proved to be able to reproduce structural size-effect in a wide range of scales and, particularly, in engineering-size problems [25].

The objective of the paper is proving that the use of an appropriate finite element technology is crucial for the study of the experimental setting and for the assessment of the results, even in the case of a very well known application, as the pullout test is. The outline of the paper is as follows. First, 
the displacement-based and the mixed strain-displacement formulations are introduced. Then, the plasticity constitutive model is presented and the Rankine yielding criterion is extended in the case of multi-axial loading condition. A regularization of the singular points, useful to avoid indetermination issues in the return mapping algorithm, is shown. Finally, numerical simulations of 2D and 3D pullout tests are presented: standard and mixed finite element analyses are compared, demonstrating both the sensibility to changes on the boundary conditions and the replication of experiments. The results show that the mixed $\varepsilon / u$ finite element provides reliable and high quality outcomes when compared to the standard irreducible formulation.

\section{Governing equations}

A solid body $\mathscr{B}$ occupying the space domain $\Omega$ is described by the position $X$ of each point with respect to a system of coordinates $x, y, z$.

On the one hand, every point of such domain has a displacement $\boldsymbol{u}$ and a total strain $\boldsymbol{\varepsilon}$. Both displacements and strains are considered small. The compatibility condition relates both fields as:

$$
-\varepsilon+\nabla^{s} u=0
$$

where $\nabla^{s}(\cdot)$ is used to denote the symmetric gradient operator. On the other hand, the equilibrium of forces in (quasi-)static conditions states that:

$$
\nabla \cdot \sigma+f=0
$$

where $\sigma$ is the Cauchy stress tensor and $f$ are the external forces applied to the body. The symbol
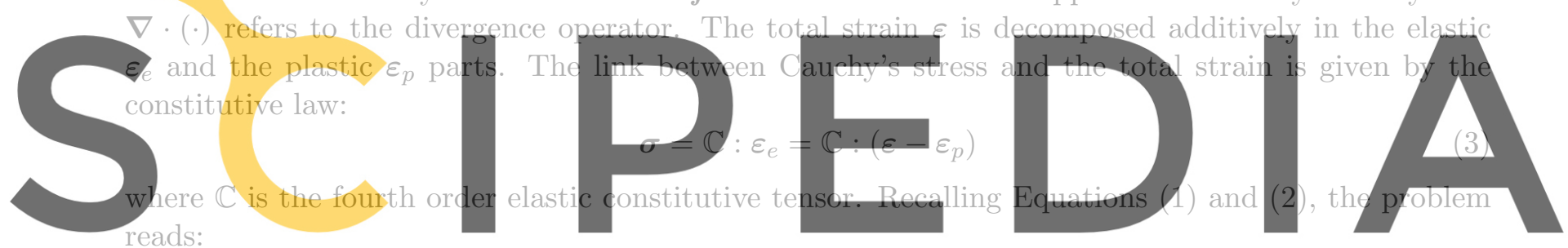

Register for free at https//www.scipedia.com to download the version without the watemark

$\nabla \cdot\left[\mathbb{C}:\left(\varepsilon-\varepsilon_{p}\right)\right]+f=0$

This set of equations represents the strong form for the mixed problem involving the unknown fields of displacements $\boldsymbol{u}$ and total strains $\varepsilon$ in the case of plasticity. In order to obtain a symmetric system, the first equation is pre-multiplied by the elastic constitutive tensor $\mathbb{C}$ :

$$
\begin{aligned}
& -\mathbb{C}: \varepsilon+\mathbb{C}: \nabla^{s} \boldsymbol{u}=\mathbf{0} \\
& \boldsymbol{\nabla} \cdot\left[\mathbb{C}:\left(\varepsilon-\varepsilon_{p}\right)\right]+\boldsymbol{f}=\mathbf{0}
\end{aligned}
$$

The irreducible problem, in terms of the displacement field $\boldsymbol{u}$ only, is recovered substituing the first equation into the second, to yield:

$$
\boldsymbol{\nabla} \cdot\left[\mathbb{C}:\left(\boldsymbol{\nabla}^{s} \boldsymbol{u}-\varepsilon_{p}\right)\right]+\boldsymbol{f}=\mathbf{0}
$$

With proper conditions on the boundary $\partial \Omega$ and evolution laws for the plastic strain field [32], both irreducible and mixed formulations provide a well posed boundary value problem.

\section{$3 \quad$ Irreducible finite elements}

Recalling the strong form in Equation (6), the corresponding weak problem can be written as:

$$
\int_{\Omega} \boldsymbol{v} \cdot\left(\boldsymbol{\nabla} \cdot\left[\mathbb{C}:\left(\boldsymbol{\nabla}^{s} \boldsymbol{u}-\boldsymbol{\varepsilon}_{p}\right)\right]\right)+\int_{\Omega} \boldsymbol{v} \cdot \boldsymbol{f}=0 \quad \forall v \in \mathbb{V}
$$


where $\mathbb{V}$ is the space of test functions which are square integrable. Integrating by parts, the forcing terms can be extracted as:

$$
\int_{\Omega} \boldsymbol{\nabla}^{s} \boldsymbol{v}: \mathbb{C}:\left(\boldsymbol{\nabla}^{s} \boldsymbol{u}-\boldsymbol{\varepsilon}_{p}\right)=F(\boldsymbol{v})
$$

where the boundary terms accounting for body forces $\boldsymbol{f}$ on $\Omega$ and tractions $\boldsymbol{t}$ on the boundary $\partial \Omega$ are collected in the term

$$
F(\boldsymbol{v})=\int_{\Omega} \boldsymbol{v} \cdot \boldsymbol{f}+\int_{\partial \Omega} \boldsymbol{v} \cdot \boldsymbol{t}
$$

The discretized version of Equation (8) is obtained by selecting a finite set of interpolation functions for the displacement field as well as the test function as:

$$
\boldsymbol{u} \rightarrow \boldsymbol{u}_{h}=\sum_{i=1}^{n_{p t s}} \boldsymbol{v}_{h}^{(i)} \boldsymbol{u}_{h}^{(i)} \quad \boldsymbol{v}_{h} \in \mathbb{V}_{h}
$$

such that the discrete functional space $\mathbb{V}_{h}$ is a subset of the continuous version $\mathbb{V} \subseteq H^{1}(\Omega)^{\operatorname{dim}}$. From Equation (8), the final discrete system of equations reads:

$$
\int_{\Omega} \nabla^{s} v_{h}: \mathbb{C}:\left(\nabla^{s} u_{h}-\varepsilon_{p}\right)=F\left(v_{h}\right)
$$

\section{For the standard finite element interpolation, linear triangles P1 and quadrilateral Q1 are considered} in this work.
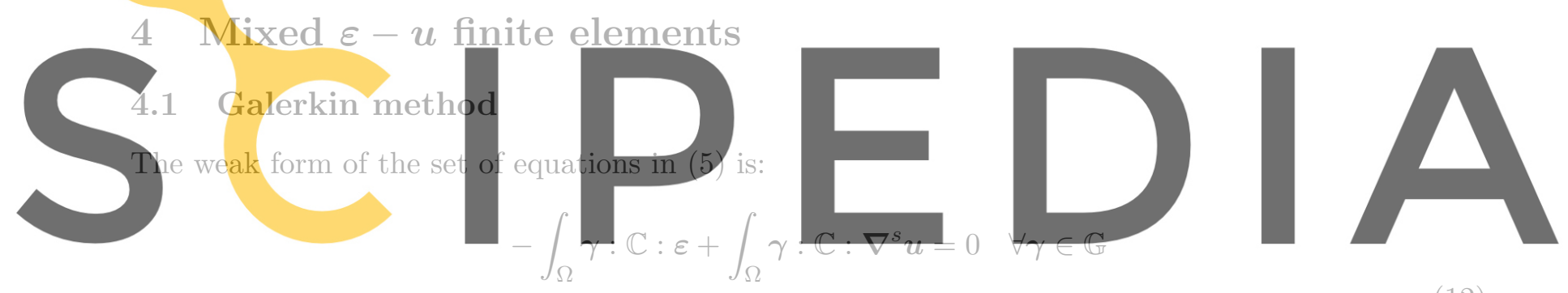

Register for free at https//www scipedia.com. to download the version without the watermark

$$
\int_{\Omega} v \cdot(\nabla \cdot \sigma)+\int_{\Omega} v \cdot f=0
$$

In this case, besides the functional space $\mathbb{V}$ for the test functions $\boldsymbol{v}$ of the displacement field $\boldsymbol{u}$, it is required to introduce the set of test function tensors for the strain $\varepsilon$ pertaining to $\mathbb{G}$. Integrating by parts the second equation, it can be written:

$$
\begin{array}{ll}
-\int_{\Omega} \gamma: \mathbb{C}: \varepsilon+\int_{\Omega} \gamma: \mathbb{C}: \nabla^{s} \boldsymbol{u}=0 & \forall \boldsymbol{\gamma} \in \mathbb{G} \\
\int_{\Omega} \boldsymbol{\nabla}^{s} \boldsymbol{v}: \boldsymbol{\sigma}=F(\boldsymbol{v}) & \forall \boldsymbol{v} \in \mathbb{V}
\end{array}
$$

From the mathematical requirements of the problem, $\mathbb{V}$ is in the space of square integrable functions $\boldsymbol{v}$ which are at least square integrable and have square integrable first derivative, whereas $\mathbb{G}$ belongs to the set of square integrable symmetric tensors $\gamma$.

The discretized version of the weak form in Equation (13) is obtained by approximating the strain tensor $\varepsilon$ and the displacement field $\boldsymbol{u}$ as

$$
\begin{gathered}
\boldsymbol{\varepsilon} \rightarrow \boldsymbol{\varepsilon}_{h}=\sum_{i=1}^{n_{p t s}} \gamma_{h}^{(i)} \varepsilon_{h}^{(i)} \quad \gamma_{h} \in \mathbb{G}_{h} \\
\boldsymbol{u} \rightarrow \boldsymbol{u}_{h}=\sum_{i=1}^{n_{p t s}} \boldsymbol{v}_{h}^{(i)} \boldsymbol{u}_{h}^{(i)} \quad \boldsymbol{v}_{h} \in \mathbb{V}_{h}
\end{gathered}
$$


The system of equations (13), in its discrete form, reads

$$
\begin{aligned}
& -\int_{\Omega} \gamma_{h}: \mathbb{C}: \varepsilon_{h}+\int_{\Omega} \gamma_{h}: \mathbb{C}: \nabla^{s} \boldsymbol{u}_{h}=0 \quad \forall \gamma_{h} \in \mathbb{G}_{h} \\
& \int_{\Omega} \boldsymbol{\nabla}^{s} \boldsymbol{v}_{h}: \boldsymbol{\sigma}=F\left(\boldsymbol{v}_{h}\right) \quad \forall \boldsymbol{v}_{h} \in \mathbb{V}_{h}
\end{aligned}
$$

For the mixed finite element method, equal interpolation finite element spaces for displacements and strains are considered in this work, and, in particular, the case of linear and bilinear interpolations, i.e. P1P1 and Q1Q1 elements. However, the stability of a discrete mixed formulation derived by the Galerkin method depends from the choice of the finite element spaces $\mathbb{G}_{h}$ and $\mathbb{V}_{h}$ as stated by the Inf-Sup condition [33]. Using equal order of interpolation does not satisfy the previous condition; consequently, a Variational Multiscale Stabilization procedure is now introduced.

\subsection{Variational Multiscale Stabilization}

The Variational Multiscale Stabilization was developed in first instance by Hughes et al. [34] and then generalized by Codina [35]. This technique modifies appropriately the variational form of the problem in order to provide the required numerical stability (see Benedetti et al. [23], Cervera et al. [26], Badia and Codina [36], Codina [37] for details).

The stabilization procedure decomposes the solution of fields $(\varepsilon, u)$ into a resolvable scale $\left(\varepsilon_{h}, \boldsymbol{u}_{h}\right)$, calculated on the FEM mesh, and an irresolvable one $(\tilde{\varepsilon}, \tilde{u})$, proper of a finer scale:

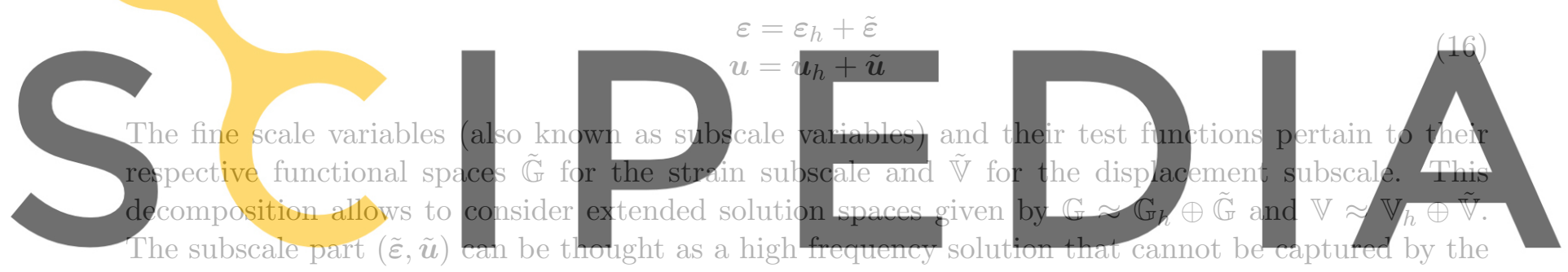

FEM mesh.

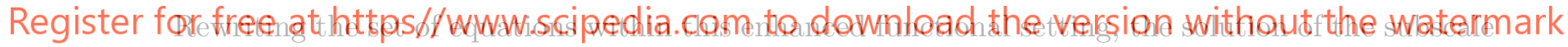

variables depends on the residuals of the strong form of the equations upon substitution of the FEM solution. Defining $\boldsymbol{R}_{1, h}$ and $\boldsymbol{R}_{2, h}$ as the residuals of the strong form equations as:

$$
\begin{aligned}
& \boldsymbol{R}_{1, h}=-\mathbb{C}: \varepsilon_{h}+\mathbb{C}: \nabla^{s} \boldsymbol{u}_{h} \\
& \boldsymbol{R}_{2, h}=\boldsymbol{\nabla} \cdot \boldsymbol{\sigma}_{h}+\boldsymbol{f}
\end{aligned}
$$

it is possible to approximate the subscale variables within each element as done in the work of Codina [35] with the projection of the residuals on the mesh grid:

$$
\begin{aligned}
& \tilde{\boldsymbol{\varepsilon}}=\tau_{\boldsymbol{\varepsilon}} \mathbb{C}^{-1}: \tilde{P}_{1}\left(\boldsymbol{R}_{1, h}\right) \\
& \tilde{\boldsymbol{u}}=\tau_{u} \tilde{P}_{2}\left(\boldsymbol{R}_{2, h}\right)
\end{aligned}
$$

where $\tilde{P}_{1}$ and $\tilde{P}_{2}$ represent the projection operators. $\tau_{u}$ and $\tau_{\varepsilon}$ are the stabilization parameters computed as:

$$
\tau_{u}=c_{u} \frac{h L_{0}}{E} \quad \text { and } \quad \tau_{\varepsilon}=c_{\varepsilon} \frac{h}{L_{0}}
$$

In the last expression, $c_{u}$ and $c_{\varepsilon}$ are arbitrary positive numbers; $E$ is the Young's modulus, being the mechanical parameter of the problem; $h$ is the representative size of the finite element mesh and $L_{0}$ is a characteristic length of the problem. To complete the stabilization method, an appropriate projection operator has to be selected in order to be able to compute the subscale variables. 


\subsubsection{OSGS stabilization}

In the Orthogonal Subgrid Scale stabilization [36], the projection operator selected to solve the unresolvable scale variables is the orthogonal projector

$$
\tilde{P}(\boldsymbol{X})=\tilde{P}_{h}^{\perp}(\boldsymbol{X})=I(\boldsymbol{X})-P_{h}(\boldsymbol{X})
$$

where $P_{h}$ represents the projection over the finite element mesh. It represents the $L^{2}$ projection of $\boldsymbol{X}$, or least square fitting, on the finite element space [38] and it is performed taking advantage of the orthogonality condition

$$
\int_{\Omega} \boldsymbol{\eta}_{h}:\left(\boldsymbol{X}_{\Pi}-\boldsymbol{X}\right)=0 \quad \forall \boldsymbol{\eta}_{h} \in \mathbb{V}_{h} \text { or } \mathbb{G}_{h}
$$

where $\boldsymbol{X}_{\Pi}$ is the projected value of $\boldsymbol{X}$ on the mesh nodes. Substituting in (18), the subscale variables $\tilde{\boldsymbol{u}}$ and $\tilde{\boldsymbol{\varepsilon}}$ can be approximated as:

$$
\begin{aligned}
& \tilde{\boldsymbol{\varepsilon}}=\tau_{\boldsymbol{\varepsilon}} \mathbb{C}^{-1}:\left[\boldsymbol{R}_{1, h}-P_{h}\left(\boldsymbol{R}_{1, h}\right)\right] \\
& \tilde{u}=\tau_{u}\left[\boldsymbol{R}_{2, h}-P_{h}\left(\boldsymbol{R}_{2, h}\right)\right]
\end{aligned}
$$

with the residuals $\boldsymbol{R}_{1, h}, \boldsymbol{R}_{2, h}$ defined in (17). Back-substituting in the set of equations of the problem, the final set of equations is:

$$
-\left(1-\tau_{\varepsilon}\right) \int_{\Omega} \gamma_{h}: \mathbb{C}:\left(\varepsilon_{h}-\nabla^{s} u_{h}\right)+
$$

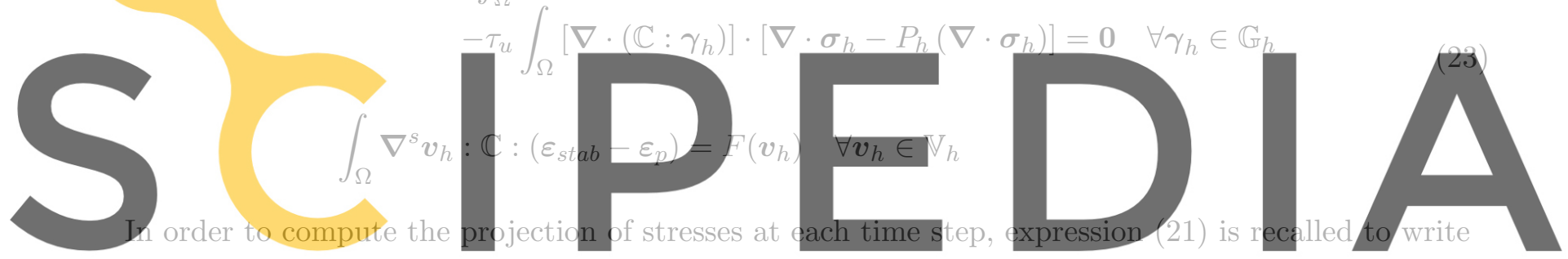

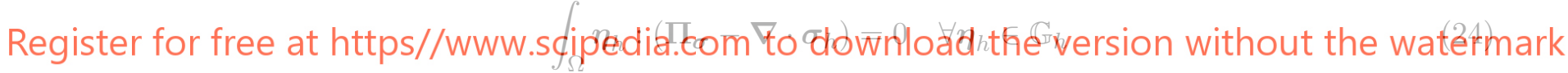

and, with the additional projection equation, it reads:

$$
\begin{aligned}
& -\left(1-\tau_{\boldsymbol{\varepsilon}}\right) \int_{\Omega} \boldsymbol{\gamma}_{h}: \mathbb{C}:\left(\boldsymbol{\varepsilon}_{h}-\boldsymbol{\nabla}^{s} \boldsymbol{u}_{h}\right) \\
& \quad-\tau_{u} \int_{\Omega}\left[\boldsymbol{\nabla} \cdot\left(\mathbb{C}: \boldsymbol{\gamma}_{h}\right)\right] \cdot\left[\boldsymbol{\nabla} \cdot \boldsymbol{\sigma}_{h}-\boldsymbol{\Pi}_{\boldsymbol{\sigma}}\right]=\mathbf{0} \quad \forall \boldsymbol{\gamma}_{h} \in \mathbb{G}_{h} \\
& \int_{\Omega} \boldsymbol{\nabla}^{s} \boldsymbol{v}_{h}: \mathbb{C}:\left[\boldsymbol{\varepsilon}_{\text {stab }}-\boldsymbol{\varepsilon}_{p}\right]=F\left(\boldsymbol{v}_{h}\right) \quad \forall \boldsymbol{v}_{h} \in \mathbb{V}_{h} \\
& \int_{\Omega} \boldsymbol{\eta}_{h}:\left(\boldsymbol{\Pi}_{\boldsymbol{\sigma}}-\boldsymbol{\nabla} \cdot \boldsymbol{\sigma}_{h}\right)=0 \quad \forall \boldsymbol{\eta}_{h} \in \mathbb{G}_{h}
\end{aligned}
$$

\subsubsection{ASGS stabilization}

In the Algebraic Subgrid Scale stabilization method [36], the projection operator is taken as the identity, that is, $\tilde{P}(\boldsymbol{X})=I(\boldsymbol{X})$ :

$$
\begin{aligned}
& \tilde{\boldsymbol{\varepsilon}}=\tau_{\boldsymbol{\varepsilon}}\left(-\boldsymbol{\varepsilon}_{h}+\boldsymbol{\nabla}^{s} \boldsymbol{u}_{h}\right) \\
& \tilde{\boldsymbol{u}}=\tau_{u}\left(\boldsymbol{\nabla} \cdot \boldsymbol{\sigma}_{h}+\boldsymbol{f}\right)
\end{aligned}
$$


Substituting in Equation (16) and then in the set of equations (15) and rearranging, the final system of equations reads:

$$
\begin{aligned}
& -\left(1-\tau_{\boldsymbol{\varepsilon}}\right) \int_{\Omega} \gamma_{h}: \mathbb{C}:\left(\boldsymbol{\varepsilon}_{h}-\boldsymbol{\nabla}^{s} \boldsymbol{u}_{h}\right) \\
& -\tau_{u} \int_{\Omega}\left[\boldsymbol{\nabla} \cdot\left(\mathbb{C}: \boldsymbol{\gamma}_{h}\right)\right] \cdot\left[\boldsymbol{\nabla} \cdot \boldsymbol{\sigma}_{h}+f\right]=0 \quad \forall \boldsymbol{\gamma}_{h} \in \mathbb{G}_{h} \\
& \int_{\Omega} \boldsymbol{\nabla}^{s} \boldsymbol{v}_{h}: \mathbb{C}:\left[\left(1-\tau_{\boldsymbol{\varepsilon}}\right) \boldsymbol{\varepsilon}_{h}+\tau_{\boldsymbol{\varepsilon}} \boldsymbol{\nabla}^{s} \boldsymbol{u}_{h}-\boldsymbol{\varepsilon}_{p}\right]=F\left(v_{h}\right) \quad \forall v_{h} \in \mathbb{V}_{h}
\end{aligned}
$$

The first term in the first equation represents a projection (smoothing) of the strain field obtained by differentiation of the discrete displacement field. This is exactly the same projection operator presented in Equation (21). The second additional term is given by the displacement subscale that, in turn, depends on the residual of the strong form of the equilibrium equation. The second equation is related to the balance of momentum. The set of equations with OSGS stabilization resembles the one for the ASGS, except for the second term in the first equation: in fact the Orthogonal Subgrid Stabilization scheme is less diffusive than the Algebraic one [39]. However, this comes at the price of solving an additional equation; in the implementation details it is shown how this problem can be circumvented.

\section{Implementation}

Both in the standard and the mixed formulations, the presence of the non-linear plastic strains $\varepsilon_{p}=$
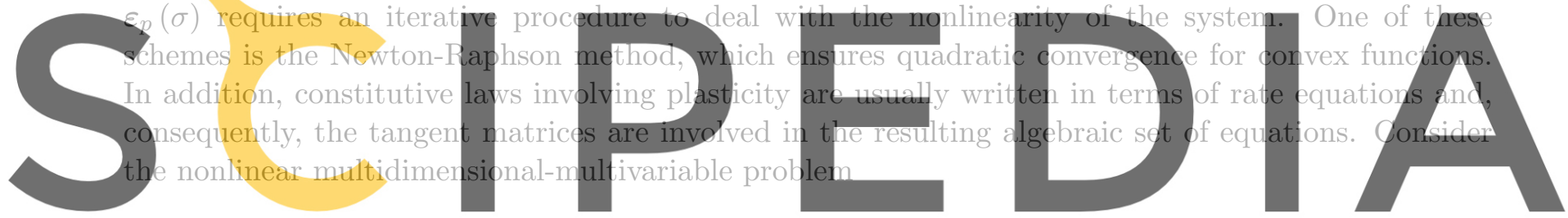

$F(X)=0$

(28)

Register for free at https//www.scipedia.com to download the version without the watermark where $X$ is the unknown. A non-linear problem of this kind can be solved starting from a Taylor approximation around the solution point at iteration $i+1$ in a particular time step $n+1$ :

$$
\boldsymbol{F}_{n+1}^{i+1} \approx \boldsymbol{F}_{n+1}^{i}+\boldsymbol{J}_{n+1}^{i} \delta \boldsymbol{X}^{i+1}
$$

where the Jacobian matrix $\boldsymbol{J}$ is defined as

$$
\boldsymbol{J}=\frac{\partial \boldsymbol{F}}{\partial \boldsymbol{X}}
$$

Assuming that $\boldsymbol{F}_{n+1}^{i+1}=0$, an iterative correction is computed as

$$
\delta \boldsymbol{X}^{i+1}=-\left[\boldsymbol{J}_{n+1}^{i}\right]^{-1} \boldsymbol{F}_{n+1}^{i}
$$

and the solution vector is updated as

$$
\boldsymbol{X}^{i+1}=\boldsymbol{X}^{i}+\delta \boldsymbol{X}^{i+1}
$$

In the irreducible formulation, the unknown vector $\boldsymbol{X}$ corresponds to the displacement field $\boldsymbol{u}$. The Jacobian matrix can be found by differentiating the set of equation with respect to the unknowns variable $\boldsymbol{u}$ at iteration $i$. In particular, it is possible to write the algebraic set of equations necessary to find the correction $\delta \boldsymbol{u}$ at the iteration $i+1$ as:

$$
\boldsymbol{K}_{t, n+1}^{i} \delta \boldsymbol{u}_{n+1}^{i+1}=\boldsymbol{F}_{n+1}^{i}
$$


where $\boldsymbol{F}$ is the residual force and the tangent stiffness matrix $\boldsymbol{K}_{t}$ is defined as

$$
\boldsymbol{K}_{t}^{i}=\boldsymbol{B}^{T} \mathbb{C}_{e p}^{i} \boldsymbol{B}
$$

being $\boldsymbol{B}$ the discrete gradient of shape functions and $\mathbb{C}_{e p}^{i}$ the algorithmic consistent elastoplastic constitutive tensor at iteration $i$.

Akin to the irreducible FEM implementation, the mixed finite element problem considers the unknown variable as $\boldsymbol{X}=[\varepsilon, \boldsymbol{u}]^{T}$. The algebraic system of equations the case of the OSGS implementation is given by

$$
-\left[\begin{array}{ccc}
\boldsymbol{M}_{\tau} & \boldsymbol{G}_{\tau} & \boldsymbol{D}_{\Pi}^{T} \\
\boldsymbol{D}_{\tau} & \boldsymbol{K}_{\tau} & \mathbf{0} \\
\boldsymbol{D}_{\Pi} & \mathbf{0} & \boldsymbol{M}_{\Pi}
\end{array}\right]_{n+1}^{i}\left[\begin{array}{c}
\delta \varepsilon_{h} \\
\delta \boldsymbol{u}_{h} \\
\delta \boldsymbol{\Pi}_{h}
\end{array}\right]_{n+1}^{i+1}=\left[\begin{array}{c}
R_{1, h} \\
R_{2, h} \\
R_{3, h}
\end{array}\right]_{n+1}^{i}
$$

where $\left(\delta \varepsilon_{h}, \delta \boldsymbol{u}_{h}, \delta \boldsymbol{\Pi}_{h}\right)$ are the iterative corrections for $\left(\varepsilon_{h}, \boldsymbol{u}_{h}, \boldsymbol{\Pi}_{h}\right)$ in the Newton-Raphson scheme. In the previous expression, $\boldsymbol{M}$ represents a mass-like projection matrix, $\boldsymbol{G}$ a gradient matrix, $\boldsymbol{D}$ a divergence matrix and $\boldsymbol{K}$ a stiffness matrix. The subscript $\tau$ refers to the fact that those matrices incorporate stabilization terms. Differentiating (25), within the hypothesis of plastic strain depending only on $\varepsilon_{h}$, the previous matrices read:

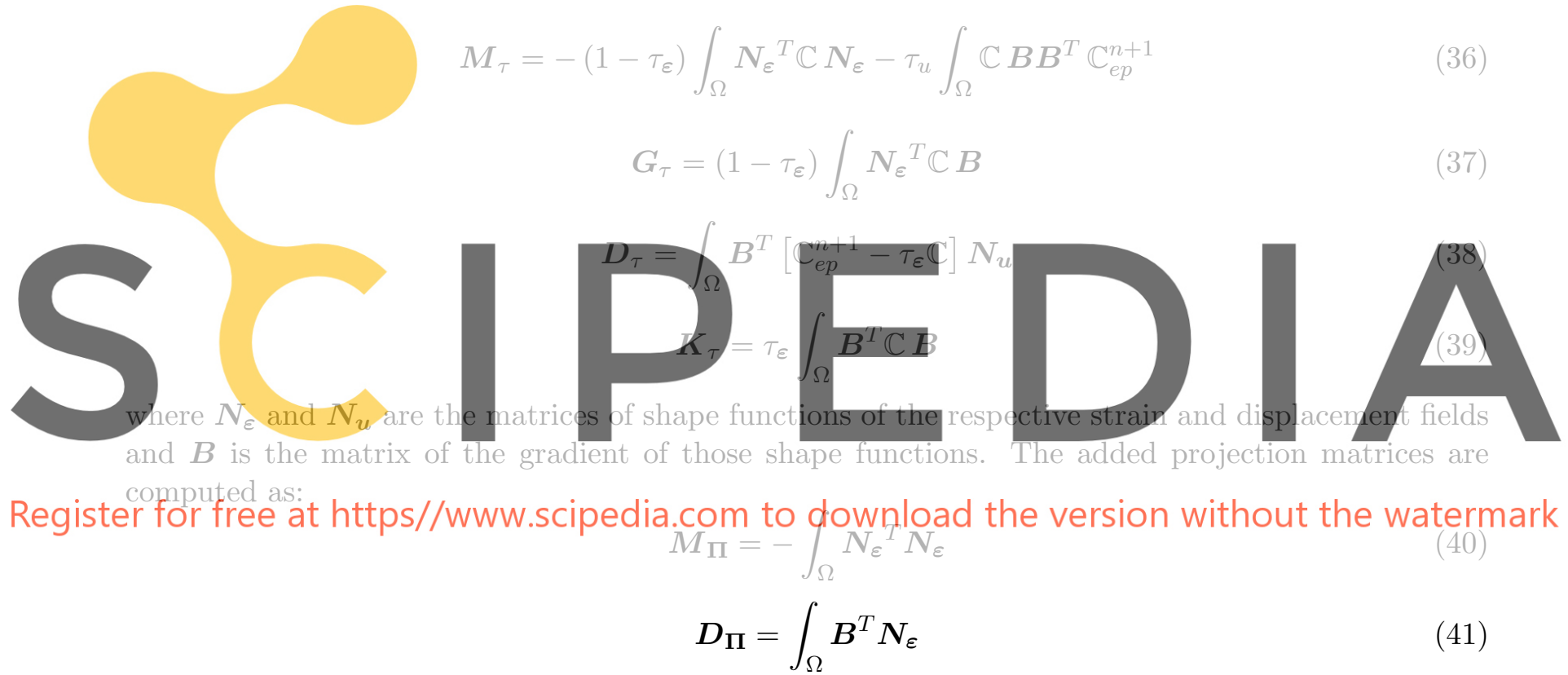

Note that the resulting algebraic system of equations is, in general, not symmetric. However, disregarding the terms due to plasticity, the system matrix is symmetric and it coincides with the one presented in Cervera et al. [26, 40]. In order to reduce computational costs, the following approximation can be considered:

$$
\boldsymbol{\Pi}_{\boldsymbol{\sigma}}^{n+1}=P_{h}^{\perp}\left(\boldsymbol{\nabla} \cdot \boldsymbol{\sigma}_{h}^{n+1}\right) \approx P_{h}^{\perp}\left(\boldsymbol{\nabla} \cdot \boldsymbol{\sigma}_{h}^{n}\right)
$$

which can be computed at the beginning of time step $n+1$.

Instead, in the case of the ASGS method, the last algebraic equation is automatically verified and the Jacobian matrix presents the mathematical structure:

$$
\boldsymbol{J}_{n+1}^{i}=\left[\begin{array}{cc}
\boldsymbol{M}_{\tau} & \boldsymbol{G}_{\tau} \\
\boldsymbol{D}_{\tau} & \boldsymbol{K}_{\tau}
\end{array}\right]_{n+1}^{i}
$$

Matrices $\boldsymbol{M}_{\tau}, \boldsymbol{G}_{\tau}, \boldsymbol{D}_{\tau}, \boldsymbol{K}_{\tau}$ are the same in both the presented methods. Details on the differentiation of the plastic strain tensor $\varepsilon_{p}$ with respect to the problem unknown $\varepsilon_{h}$ are given in the work by Benedetti et al. [23]. 


\section{Rankine plasticity model}

The Rankine yielding criterion is defined by taking the i-th principal stress, denoted with $\sigma^{i}$, and comparing it to the threshold limit stress $r(\xi)$ :

$$
f(\boldsymbol{\sigma}, q)=\sigma^{i}-r(\xi)
$$

where $r(\xi)$ is

$$
r(\xi)=\sigma_{y}-q(\xi)
$$

being $\sigma_{y}$ the uniaxial yielding value of stresses and $q(\xi)$ the hardening/softening function. In plasticity, the Cauchy's stress tensor is defined as:

$$
\boldsymbol{\sigma}=\mathbb{C}: \varepsilon_{e}=\mathbb{C}:\left(\varepsilon-\varepsilon_{p}\right)
$$

where the total strain tensor $\varepsilon$ can be split in elastic strain $\varepsilon_{e}$ and plastic strain $\varepsilon_{p}$. The plastic strain-like database is composed of the tensor $\varepsilon_{p}$ and the isotropic hardening strain-like variable $\xi$. The evolution equations, also known as flow rule, are defined as:

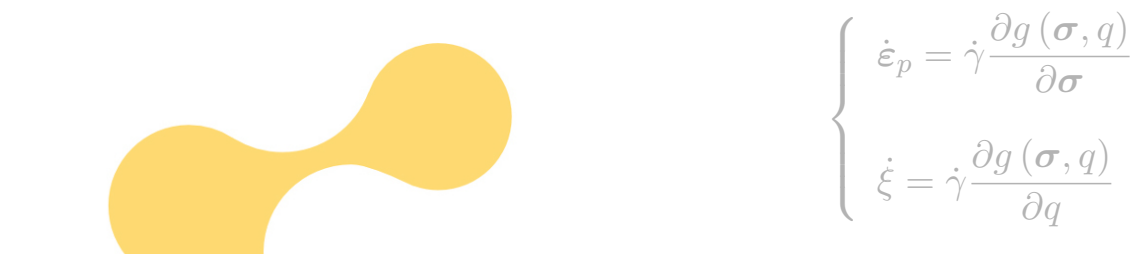

where $\dot{\gamma}$ is the plastic multiplier and the function $g(\sigma, q)$ represents the plastic potential. In associated plasticity, the plastic poten in Such definition of the construction. In the case

depends on the stress
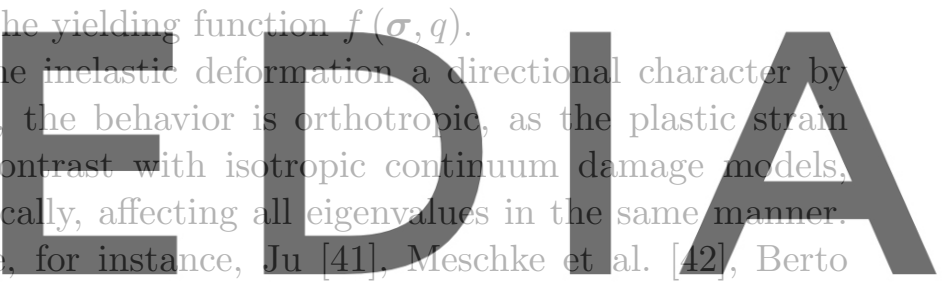

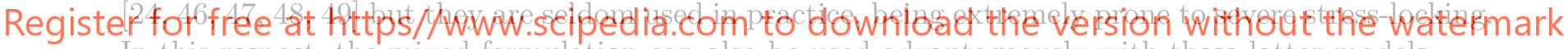

In this respect, the mixed formulation can also be used advantageously with these latter models.

In the Haig-Westergaard space, the Rankine criterion appears as the intersection of three planes

in the first octant, where $\sigma^{1}, \sigma^{2}, \sigma^{3}$ are positive (Figure 1). The yielding surface loci are also described by the following conditions:

$$
\begin{aligned}
& \text { Faces: } \sigma^{1}=r(\xi) \text { or } \sigma^{2}=r(\xi) \text { or } \sigma^{3}=r(\xi) \\
& \text { Edges: } \sigma^{1}=\sigma^{2}=r(\xi) \text { or } \sigma^{2}=\sigma^{3}=r(\xi) \text { or } \sigma^{1}=\sigma^{3}=r(\xi) \\
& \text { Apex: } \sigma^{1}=\sigma^{2}=\sigma^{3}=r(\xi)
\end{aligned}
$$

The edges and apex are singular cases that require special treatment, to avoid ill-definition of the flow direction in the case of associated plasticity [50]. One possibility to circumvent this problem is to smooth the domain in the vicinity of the corners [51, 52].

The sharp edges are regularized with a quarter of cylindrical surface whereas the apex with an eighth of a sphere. In this way, it is possible to join up the three faces in a unique surface, whose radii are expressed as fraction $c \in[0,1]$ of the uniaxial threshold:

$$
\rho(c, q(\xi))=c\left(\sigma_{y}-q(\xi)\right)
$$

The Extended Rankine yielding criterion is defined as:

$$
f(c, \boldsymbol{\sigma}, q(\xi))=\sqrt{\left\langle f_{1}(c, \boldsymbol{\sigma}, q(\xi))\right\rangle^{2}+\left\langle f_{2}(c, \boldsymbol{\sigma}, q(\xi))\right\rangle^{2}+\left\langle f_{3}(c, \boldsymbol{\sigma}, q(\xi))\right\rangle^{2}}-\rho(c, q(\xi))
$$




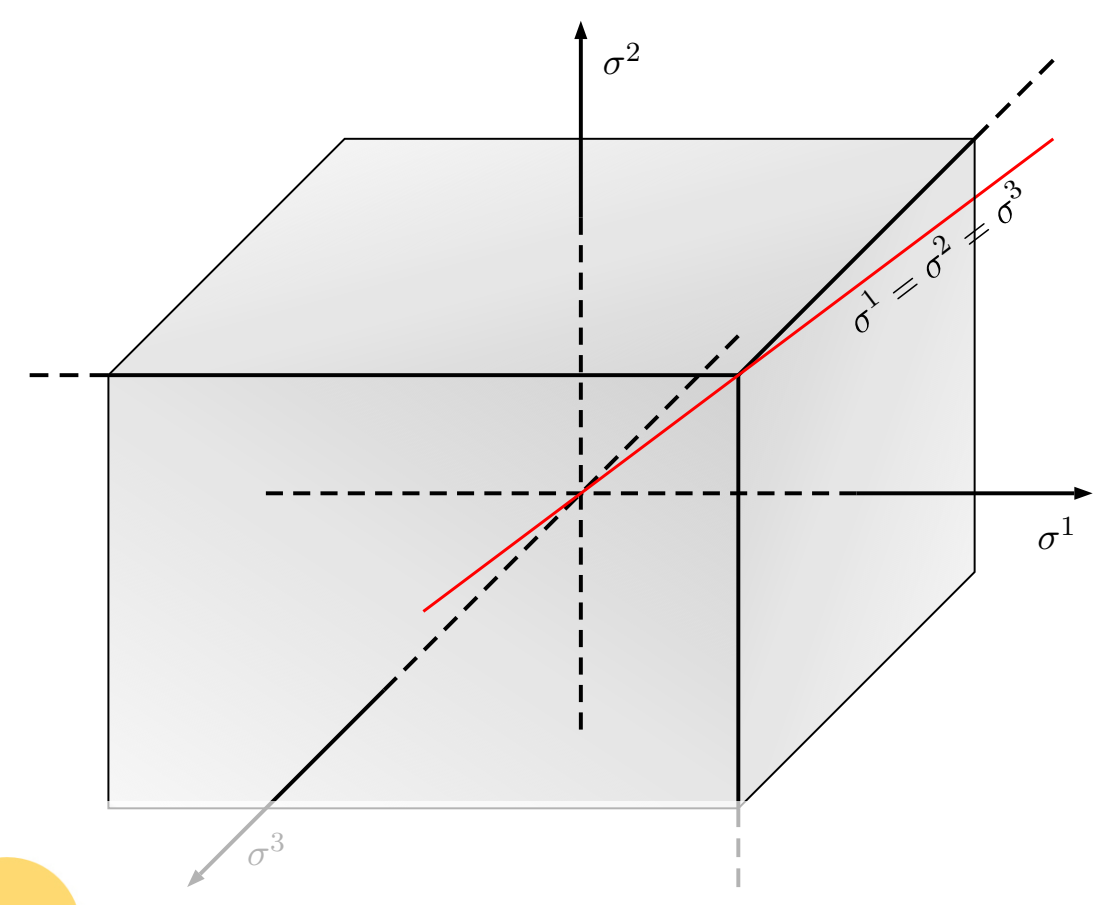

Figure 1: Representation of the yielding surface in the principal stress space $\left(\sigma^{1}, \sigma^{2}, \sigma^{3}\right)$ for $c=0$.

The gray area is the admissible stress domain, while the red line represents the hydrostatic axis.

where $f_{i}(c, \sigma, q(\xi))$ is the uniaxial yield function defined as:

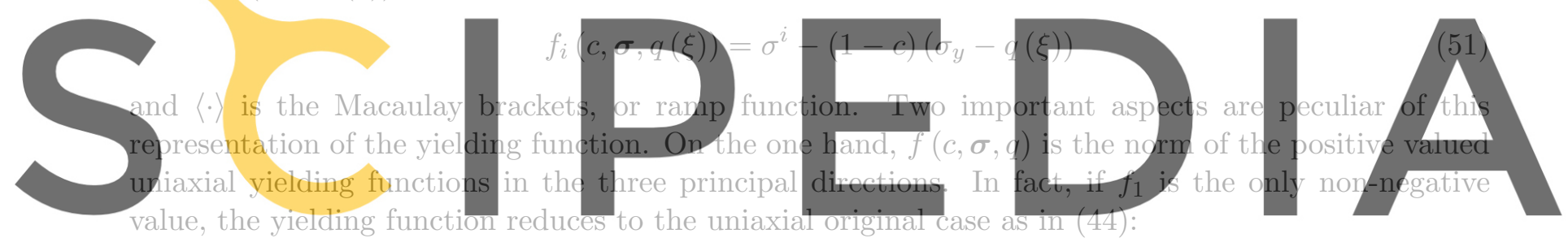

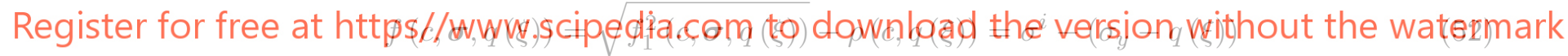

On the other hand, the surface is smooth and differentiable at all points. In the case of $c=0$ the original yielding surface with sharp edges is recovered, whereas, when $c=1$, the first octant presents a spherical sector.

Defining:

$$
\bar{f}_{i}(\boldsymbol{\sigma}, q)=\frac{\left\langle f_{i}(\boldsymbol{\sigma}, q)\right\rangle}{\sqrt{f_{1}(\boldsymbol{\sigma}, q)^{2}+f_{2}(\boldsymbol{\sigma}, q)^{2}+f_{3}(\boldsymbol{\sigma}, q)^{2}}}
$$

the evolution equations for the continuous case can be compactly written as:

$$
\left\{\begin{array}{l}
\dot{\boldsymbol{\varepsilon}}_{p}=\dot{\gamma} \sum_{i=1}^{3} \bar{f}_{i}(\boldsymbol{\sigma}, q) \mathbb{H}^{i i} \\
\dot{\xi}=\dot{\gamma}\left(c+(1-c) \sum_{i=1}^{3} \bar{f}_{i}(\boldsymbol{\sigma}, q)\right)
\end{array}\right.
$$

where the second order tensor $\mathbb{H}^{i j}=\phi^{i} \otimes \phi^{j}$ represents the direction of the plastic flow, being $\phi^{j}$ the $\mathrm{j}$-th eigenvector of the stress tensor.

The time variation of the plastic multiplier is computed as [32]:

$$
\dot{\gamma}=\frac{\frac{\partial f}{\partial \boldsymbol{\sigma}}: \mathbb{C}: \dot{\boldsymbol{\varepsilon}}}{\frac{\partial f}{\partial \boldsymbol{\sigma}}: \mathbb{C}: \frac{\partial f}{\partial \boldsymbol{\sigma}}-\frac{\partial f}{\partial q} \frac{\partial q}{\partial \xi} \frac{\partial f}{\partial q}}
$$




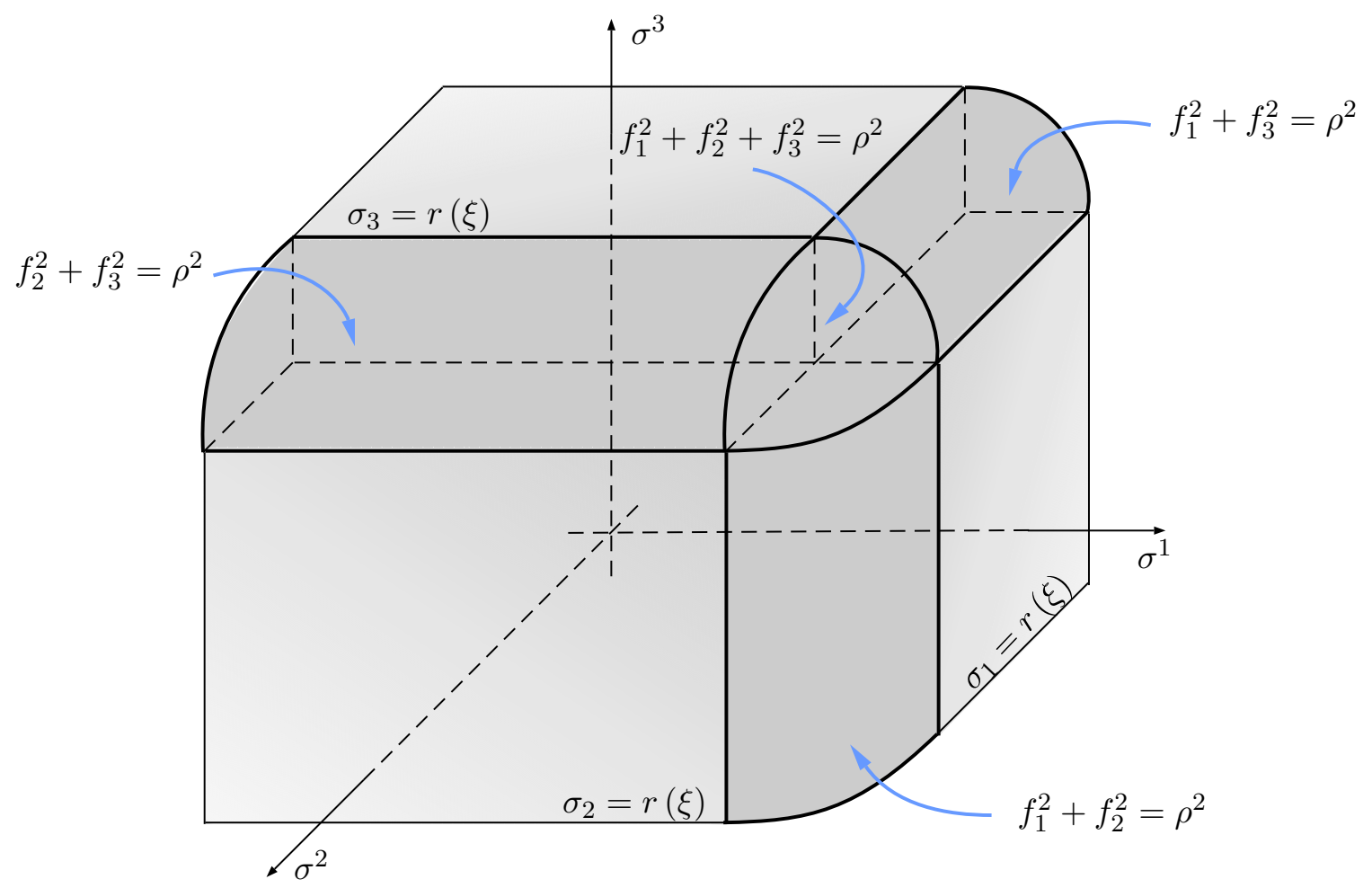

Figure 2: Extended Rankine yield surface: the smoothing of the corners is highlighted and each part presents its own descriptive equation

The discrete version of Rankine plasticity model follows the closest point projection algorithm, where introducing a Backward Euler time scheme, the plastic multiplier $\Delta \gamma^{n+1}$ is computed by finding the root of the yield function at the step $n+1$ :

$$
\begin{aligned}
& f^{n+1}\left(\boldsymbol{\sigma}^{n+1}, q^{n+1}\right)=\sqrt{f_{1}\left(\boldsymbol{\sigma}^{n+1}, q^{n+1}\right)^{2}+f_{2}\left(\boldsymbol{\sigma}^{n+1}, q^{n+1}\right)^{2}+f_{3}\left(\boldsymbol{\sigma}^{n+1}, q^{n+1}\right)^{2}}-\rho(q)= \\
& =\left[\sum_{i=1}^{n_{\text {ret }}}\left(\mathbb{H}^{i}: \boldsymbol{\sigma}_{\text {trial }}-\Delta \gamma^{n+1} \mathbb{H}^{i} \mathbb{C} \frac{\partial f^{n+1}}{\partial \boldsymbol{\sigma}^{n+1}}-(1-c)\left(\sigma_{y}-q\left(\xi^{n}+\Delta \gamma^{n+1} \frac{\partial f^{n+1}}{\partial q^{n+1}}\right)\right)\right)^{2}\right]^{\frac{1}{2}} \\
& -c\left(\sigma_{y}-q\left(\xi^{n}+\Delta \gamma^{n+1} \frac{\partial f^{n+1}}{\partial q^{n+1}}\right)\right)=0
\end{aligned}
$$

where $n_{\text {ret }}$ is the number of principal stresses to be considered in the return mapping. For linear hardening/softening, function $q(\xi)$ reads

$$
q(\xi)=\left\{\begin{array}{lll}
\frac{H_{S}}{\sigma_{y}} \xi & \text { for } & 0 \leq \xi \leq \frac{\sigma_{y}}{H_{S}} \\
0 & \text { for } & \frac{\sigma_{y}}{H_{S}} \leq \xi \leq \infty
\end{array}\right.
$$

and the value of $\Delta \gamma^{n+1}$ is explicitly computed from Equation (56). For exponential softening, $q(\xi)$ is

$$
q(\xi)=\sigma_{y}\left[1-\exp \left(\frac{-2 H_{S}}{\sigma_{y}} \xi\right)\right] \quad \text { for } \quad 0 \leq \xi \leq \infty
$$

and a local Newton-Raphson iterative scheme is required to compute $\Delta \gamma^{n+1}$. The update of the stress and the hardening function is performed as:

$$
\left\{\begin{array}{l}
\boldsymbol{\sigma}^{n+1}=\mathbb{C}:\left[\varepsilon^{n+1}-\varepsilon_{p}^{n+1}\right]=\boldsymbol{\sigma}_{\text {trial }}^{n+1}-\mathbb{C}: \Delta \gamma^{n+1} \frac{\partial f^{n+1}}{\partial \boldsymbol{\sigma}^{n+1}} \\
q\left(\xi^{n+1}\right)=q\left(\xi^{n}+\Delta \gamma^{n+1} \frac{\partial f^{n+1}}{\partial q^{n+1}}\right)
\end{array}\right.
$$


Following standard arguments [32], the continuous elastoplastic constitutive fourth order tensor is:

$$
\mathbb{C}^{e p}=\left[\mathbb{C}-\frac{\left(\mathbb{C}: \frac{\partial f}{\partial \boldsymbol{\sigma}}\right) \otimes\left(\mathbb{C}: \frac{\partial f}{\partial \boldsymbol{\sigma}}\right)}{\frac{\partial f}{\partial \boldsymbol{\sigma}}: \mathbb{C}: \frac{\partial f}{\partial \boldsymbol{\sigma}}-\frac{\partial f}{\partial q} \frac{\partial q}{\partial \xi} \frac{\partial f}{\partial q}}\right]
$$

In the discrete time setting, an algorithmic tangent operator is used; this is defined by the change of stresses around an admissible equilibrium position (i.e. $\boldsymbol{\sigma}^{n+1}$ ) due to a small variation in the strain. The i-th column of the approximated constitutive matrix can be computed as:

$$
\left.\mathbb{C}_{e p}^{n+1} \hat{\boldsymbol{e}}_{i} \approx \frac{\boldsymbol{\sigma}\left(\varepsilon+h \hat{\boldsymbol{e}}_{i}\right)-\boldsymbol{\sigma}\left(\varepsilon-h \hat{\boldsymbol{e}}_{i}\right)}{2 h}\right|_{n+1}
$$

where $\hat{\boldsymbol{e}}_{i}$ is the unit vector of the i-th direction in the reference system. The smaller the perturbation, the closer the approximation tends to the original definition of the elastoplastic tensor. However, it has to be noted that the lower limit of the value of the perturbation is proportional to the machine precision, below which numerical cancellation and instabilities are frequent. Numerical experiments justify a perturbation size of $h=10^{-5}\|\varepsilon\|$. First, from the value $\varepsilon^{n+1}$, the perturbed strain tensors $\varepsilon+h \hat{\boldsymbol{e}}_{i}$ and $\varepsilon-h \hat{\boldsymbol{e}}_{i}$ in the selected direction are computed. Second, the respective stress values are evaluated and the return mapping procedure is applied on both of them. Finally, the i-th column of $\mathbb{C}_{e p}^{n+1}$ can be approximated as in Equation (61). Further details and alternative approximation schemes can be found in the work by Miehe [53], Pérez-Foguet et al. [54], Reps and Rall [55].

\section{Softening behavior}

In a softening process, the energy dissipated by inelastic behavior is linked with the fracture energy $G_{f}[56]$, defined by unit surface. When using a plastic model defined in terms of stress and strain, the dissipated plastic energy $\mathscr{W}_{p}$ is defined by unit volume. In the discrete FE setting, these two definitions are related through a characteristic length $l_{c h}$, connected to the mesh resolution:

$$
\mathscr{W}_{p}=\frac{G_{f}}{l_{c h}}
$$

On the one hand, this relation enforces mesh-size objectivity of the FE results, and, on the other end, it effectively introduces structural size effect in quasi brittle failure [25]. In the plastic model, recovering Equation (46) and (54), the rate of plastic work is computed as:

$$
\dot{\mathscr{W}}_{p}=\boldsymbol{\sigma}: \dot{\varepsilon}_{p}=r(\xi) \dot{\xi}
$$

From expressions (57) and (58), in both the linear and exponential softening cases, the total plastic work is calculated then as:

$$
\mathscr{W}_{p}=\int_{t=0}^{t=\infty} \dot{\mathscr{W}}_{p} \mathrm{~d} t=\int_{\xi=0}^{\xi=\infty} r(\xi) \dot{\xi}=\frac{\sigma_{y}^{2}}{2 H_{S}}
$$

and this represent the area underlying the $r-\xi$ curve. Now, comparing expressions (62) and (64), the parameter $H_{S}$ can be computed as:

$$
H_{S}=\frac{\sigma_{y}^{2}}{2 G_{f}} l_{c h}=\bar{H}_{S} l_{c h}
$$

The parameter $\bar{H}_{S}$, depends only on material properties, whereas $l_{c h}$ depends on the resolution of the discretization. In fact, the size of the strain concentration band depends on the finite element technology used, as pointed out by Cervera et al. [26]: irreducible finite elements, due to the discontinuous strain field, provide a concentration band within a single element span whereas in the $\varepsilon-u$ mixed FE formulation, with inter-elemental continuous strain, the slip line spans two elements. Consequently, the characteristic length $l_{c h}$ is taken accordingly. 


\section{Numerical Simulations}

In the present section, the problem of pullout test of a metal anchor in a concrete block is addressed, both in 2D and in 3D. The aim of the pullout test is to evaluate the overall response of embedded steel heads in concrete. On the one hand, the experiment provides valuable data about the fracture pattern and the quality of the anchorage. On the other hand, the maximum tensile strength and the post peak energy dissipation of the concrete can be evaluated with simplified or empiric formulas.

The objective of the following numerical simulations is to demonstrate the advantages of the $\varepsilon / u$ mixed FEM with respect to the standard irreducible one. These are assessed in two complementary aspects: (a) convergence to the appropriate solution, without spurious numerical issues, like meshsize and mesh-bias dependence; (b) enhanced accuracy, able to reproduce optimally the solution for a computational mesh of a chosen degree of refinement. These two facts translate in a high-fidelity simulation of experimental results obtained for the pullout test, in a way that makes it possible to study the sensibility of the data to the variations, desired or not, of the boundary conditions or the bracing system.

Computations have been realized using an enhanced version of COMET-Coupled mechanical and thermal analysis [57], developed by the authors at the International Center of Numerical Methods in Engineering (CIMNE) in Barcelona, Spain. The geometrical models have been created using GiD, a pre and post-processing software, also developed by CIMNE. In all the examples, the convergence tolerance used for the iterative Newton-Raphson procedure is $10^{-4}$ with respect to the residual forces.

\subsection{D Pullout Test}

The 2D pullout test under consideration was performed by Dejori [18], later published by Thenier and Hofstetter [19]. The test consists of a panel of unreinforced concrete of dimensions $700 \times 500 \mathrm{~mm}^{2}$ in which a T-shaped steel flange is embedded. The latter is $80 \times 20 \mathrm{~mm}^{2}$ in the horizontal part, whereas the vertical stem has a width of $10 \mathrm{~mm}$ (Figure 3).

The experiment, shown in Figure 4(a), is carried out by imposing a vertical displacement to the steel component, which translates in a force applied to the concrete in the surrounding of the horizontal flange. The consequent displacement/reaction plot of the structure is recorded until failure is reached. In order to apply such force to the specimen without suffering of rigid body motions, a bracing system is devised on the sides of the steel flange, as shown in Figure 4(b).

Multiple setups of this experiment are reported, in terms of embedding depth and bracing position. On the one hand, the steel head is positioned at three different positions (60, 80 and $100 \mathrm{~mm}$ ) from the top surface of the concrete panel. In the present numerical simulations, only the latter case of 100 $\mathrm{mm}$ is considered, following the work by Thenier and Hofstetter [19]. On the other hand, the bracing system presents two different dispositions. The first one considers the steel rods centered at a distance of $70 \mathrm{~mm}$ from the sides. The rods are then connected with a flange of width of $50 \mathrm{~mm}$ in contact with the top of the panel. Instead, the second setup is reported to reduce the distance of between the two bracing systems: "The distance between the vertical supports has been shortened in order to have the expected fracture growing in the direction of the supports at an average inclination of 30 degrees" [18]. Despite explicit values are not given, the distance of the bracing system to the symmetry axis can be computed as

$$
l_{b r}=\frac{w_{f}}{2}+\frac{\sqrt{3}}{2} h_{e f}
$$

where $w_{f}$ is the flange width equal to $80 \mathrm{~mm}$ and $h_{e f}$ is the embedding height. Consequently, $l_{b r}$ has a value of about $92,109,126 \mathrm{~mm}$ with respect to the $60,80,100 \mathrm{~mm}$ deep setups. Additionally, the $50 \mathrm{~mm}$ strip has been substituted by a metal bearing in order to concentrate the load.

Although the experiment appears to be quite straightforward in its description, significant differences and unexpected results were reported. Almost independently of the flange depth,it is possible to identify two families of failure modes in the experimental series. The first one is quasi-symmetric, which implies that the load has been evenly transferred from the steel flange to the specimen. Surely, a small amount of sway in the fractures is to be expected due to the accidental misalignment of the 


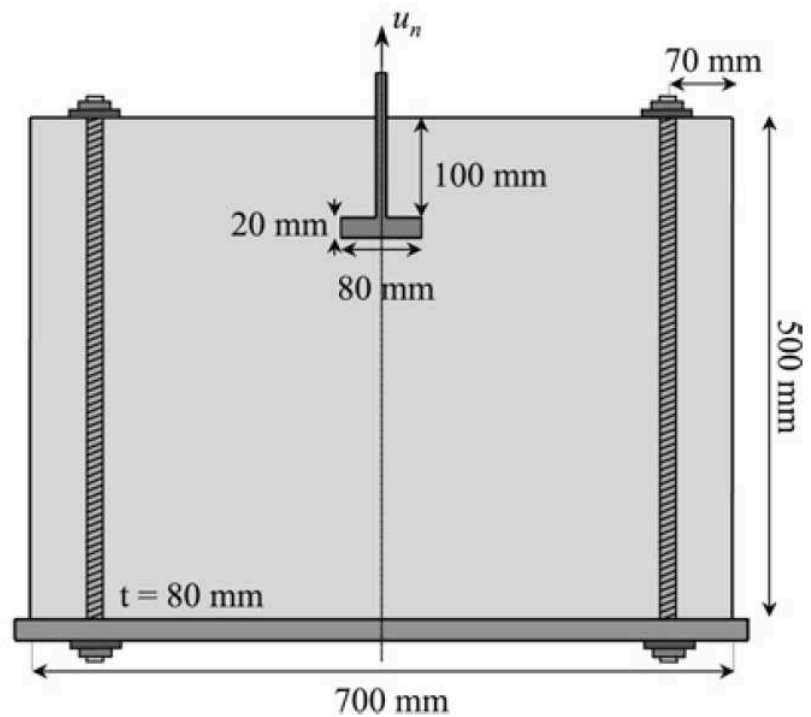

Figure 3: Geometry of the 2D pullout test (from Thenier and Hofstetter [19])

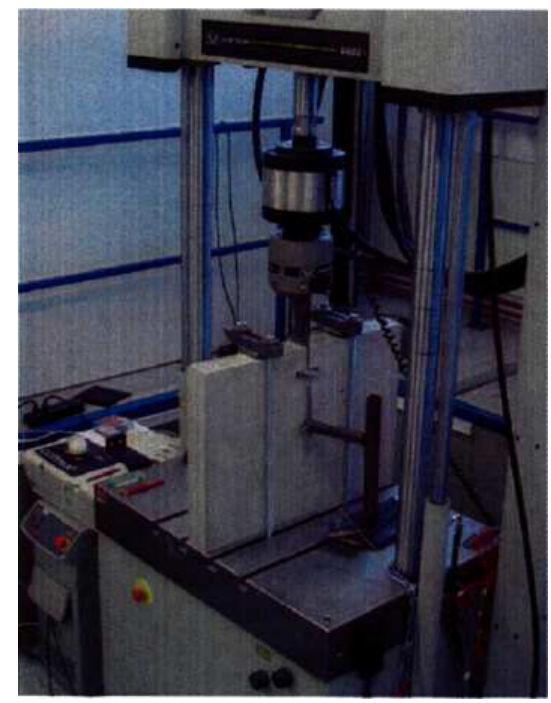

(a)

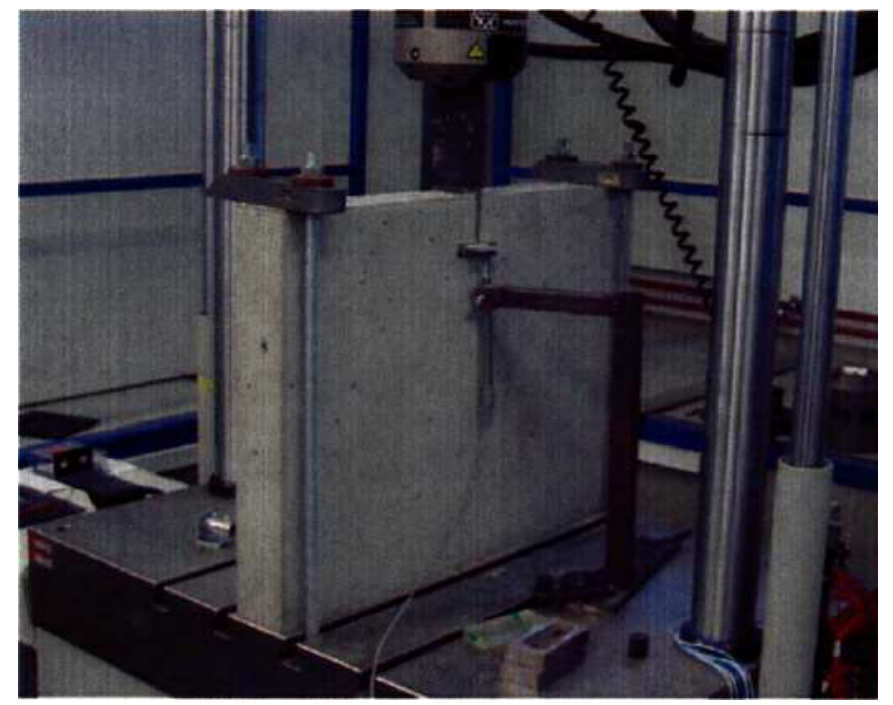

(b)

Figure 4: Detail of the experimental setup and the two different bracing systems used in the 2D pullout test $[18]$ 
load, as well as the intrinsic heterogeneity of concrete. The symmetric behaviour, even if it is not the more frequent, happens for all the setup configurations (Figure 5). The second family of failure modes

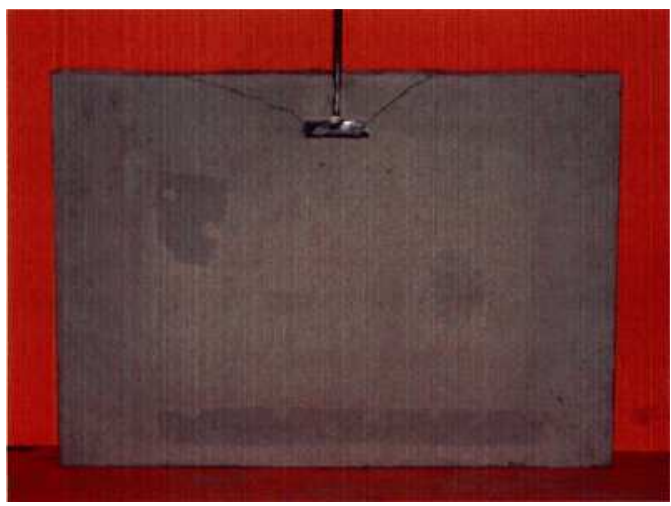

(a)

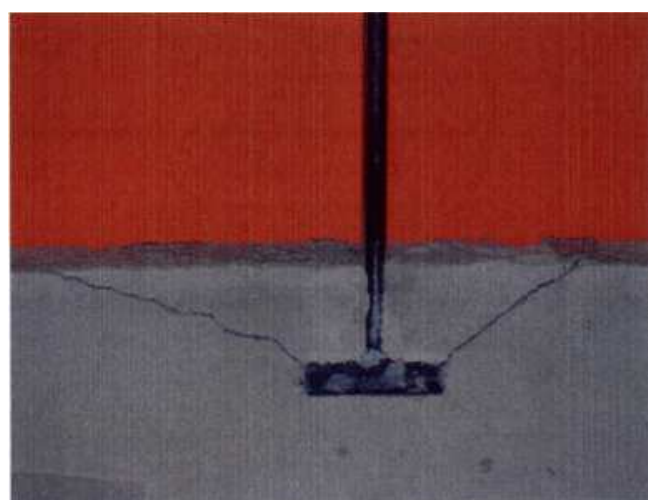

(b)

Figure 5: Detail of the symmetric results for the 2D pullout test in the case of 6 centimeters embed [18]

is clearly asymmetric. Whilst on one side of the specimen a single fracture line forms and progresses upwards to the upper surface (Figure 6 and Figure 7), on the other side a peculiar bifurcation behavior appears, with fracture lines that reach the upper and lower part of the concrete panel. It is unclear from the reported experimental data if the asymmetry is due to different lateral boundary conditions or a sudden change in the force configuration during the test.

The first setup, characterized by spread bracing, results very frequently in the asymmetric mode of failure, with an almost straight crack growing in the direction of the upper bearing whereas the bifurcation appears around the half-height of the panel, independently of the flange depth (Figure 6).

As far as the second setup is concerned, the effect of the closer bracing as well as of concentrating the load with different bearings is evident (Figure 7). The fracture lines form closer to the steel head and show a more symmetrical behavior. Nevertheless, the bifurcation pattern appears again, always on the side with a more curved crack.

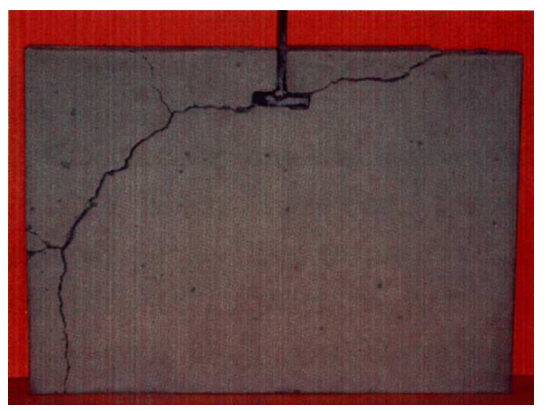

(a)

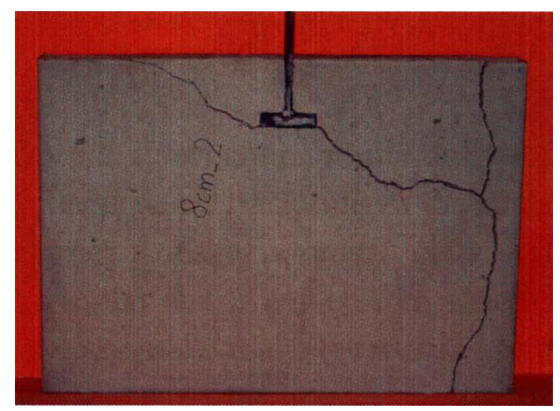

(b)

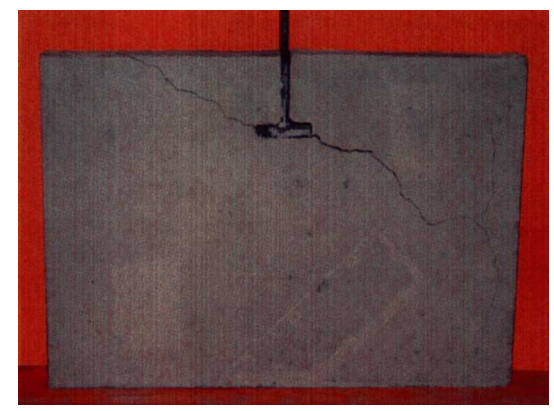

(c)

Figure 6: Asymmetric results for the first experimental setup (distant bracing) [18]

It is clear from this that the distance of the bracing frame plays a major role in the outcome of the experiment, as well as the concentration of stresses under the bearings. Not so clear is the dependence of the test results on the mechanical characteristics of the rods of the bracing system, which provide a different stress state for a similar deformation field. With or without little bracing, the concrete panel may be lifted from its base. In addition, little attention has been given to the effect of the sequence of cracking. The delay in the formation of cracks could be the main cause of asymmetry in the experimental outcomes. In fact, if a fracture has already appeared on one side of the panel, the pullout of the anchor provokes a shift of the force scheme resulting in a bending moment in the concrete (Figure 8). This fact would easily explain the onset of horizontal tension which results in 


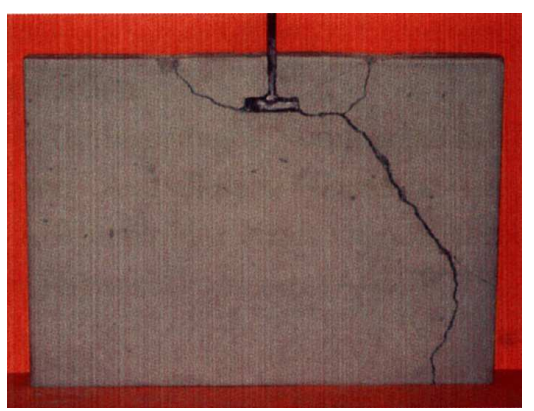

(a)

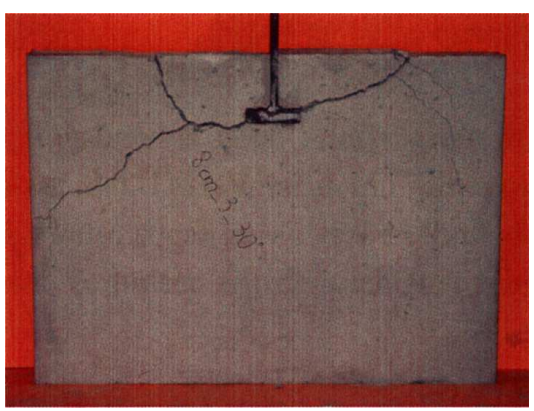

(b)

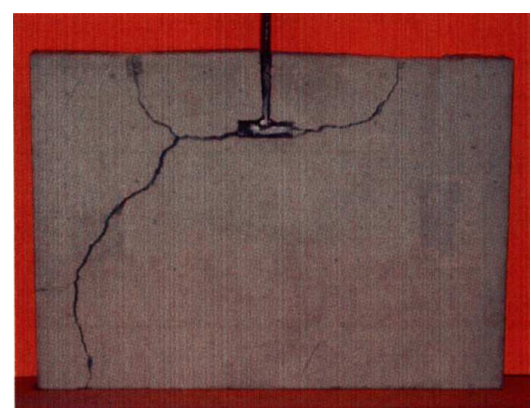

(c)

Figure 7: Asymmetric results for the second experimental setup (close bracing) [18]

vertical cracking.
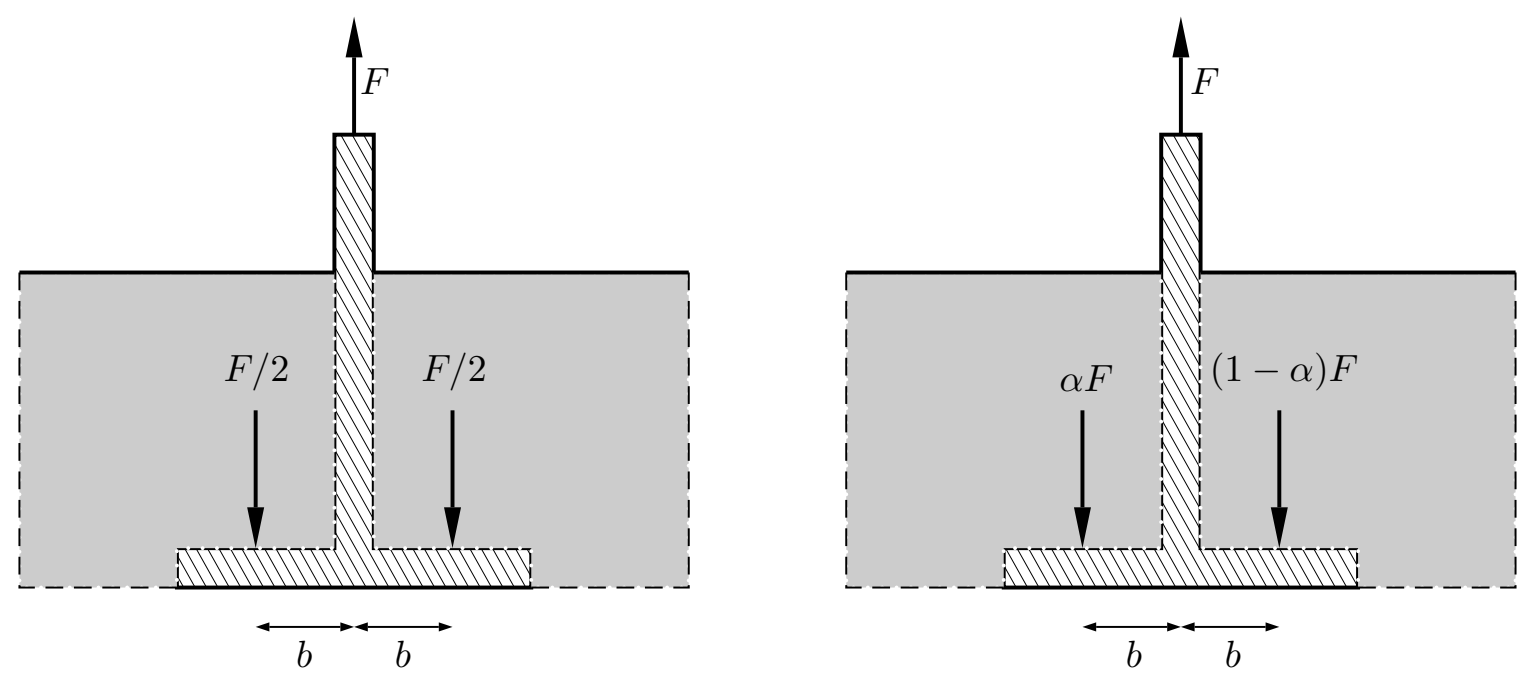

Figure 8: Symmetric and asymmetric force configuration for the pullout test. On the right, the symmetric case due to the initial uncracked state of the concrete. On the left, when one of the sides is cracked, the force shifts to the other side of the specimen and a bending moment equivalent to $(1-\alpha) 2 F b$ appears $(\alpha \leq 1 / 2)$.

In the following, two different case studies are presented. The first one is a pure shear case, where a (simplified) analytical solution is compared to the finite element one, to assess the correctness of the numerical results. In the second one, the experimental test cases are addressed and the dependency of the results on the steel bracing constraint is studied.

In all the examples, the discretized domain represents half of the concrete panel, taking advantage of the vertical symmetry line (Figure 9). As shown in the picture, the steel bracing and the top constraint are modeled as a strip of elements which connects line FG in the upper surface to the line $\mathrm{CB}$ in the bottom interface. No other connection or friction is simulated between the bracing system and the concrete panel. The thickness of the strip coincides with the resisting area of the metal rods. In this way, it is possible to simulate the presence of a vertical elastic constraint, but allowing horizontal sliding. The pullout of the steel flange is modeled imposing the vertical displacement of the boundary HILM in contact with the head. The complete computational mesh consists of a structured grid with 6936 quadrilateral elements and 7105 nodes. The characteristic size of the concrete element is $5 \mathrm{~mm}$.

The mechanical properties for the concrete are taken from the cited references. Young's modulus $E=36630 \mathrm{MPa}$ with a Poisson's ratio $\nu=0.2$. The tensile strength $\sigma_{y}=3780 \mathrm{kPa}$ and the fracture energy $\mathscr{G}_{f}=65 \mathrm{~J} / \mathrm{m}$. 


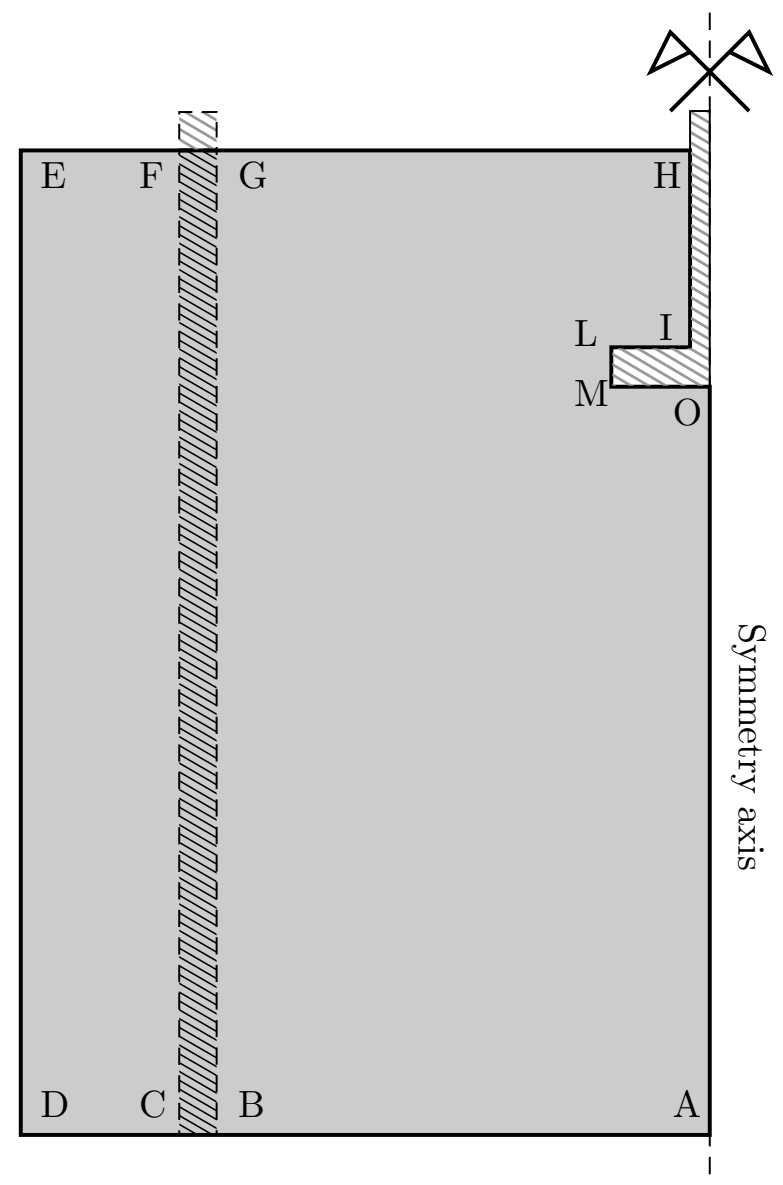

(a)

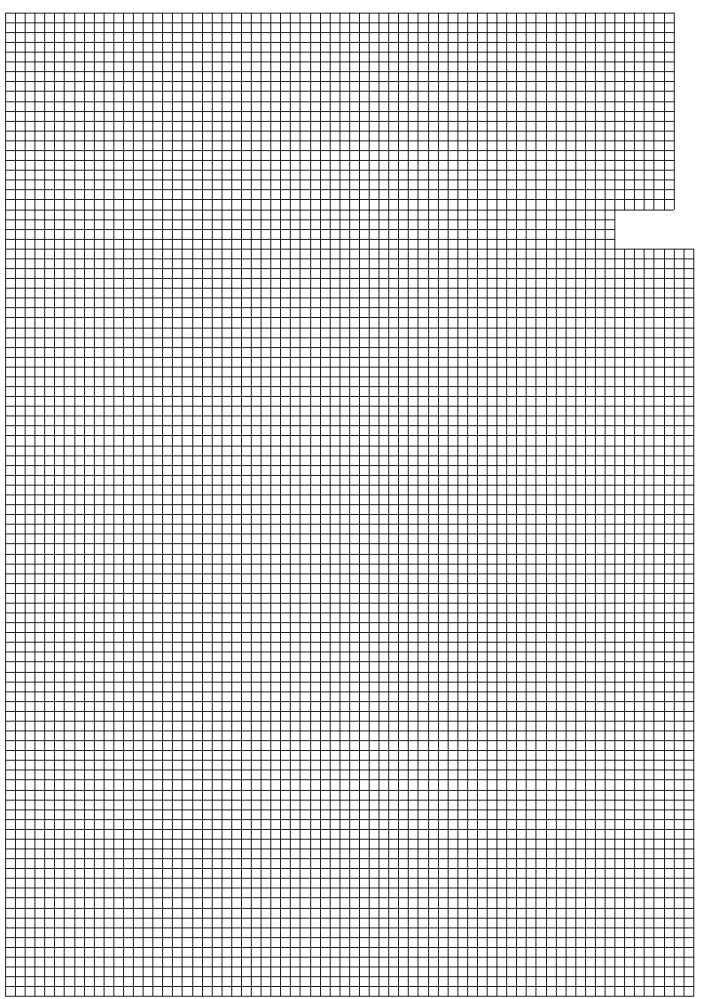

(b)

Figure 9: Discretization of the 2D pullout problem: (a) the discretized domain and (b) the structured mesh used in the analysis for the concrete panel. 


\subsubsection{Virtual shear test}

In the first example, the boundary HILM, representing the contact with the steel flange, is constrained to move vertically, but no horizontal restriction is set. Considering the upper part of the panel as a semi infinite strip as in Figure 10, the imposed displacement $u_{0}$ induces a tensional state of pure shear, with average value along the line LL' $\tau=F / A$. This approximation holds for ML/ML' $>>1$. Locally, a concentration of stresses appears close to the imposed boundary condition (Figure 11(a)). The elastic stresses computed in the vicinity of point $M$ are shown in Figure 11(b): the principal directions are at $45^{\circ}$ with respect to the horizontal, with equal value and opposite sign of compression and tension. Considering a quasi-brittle material, with a Rankine yielding criterion, the initiation of

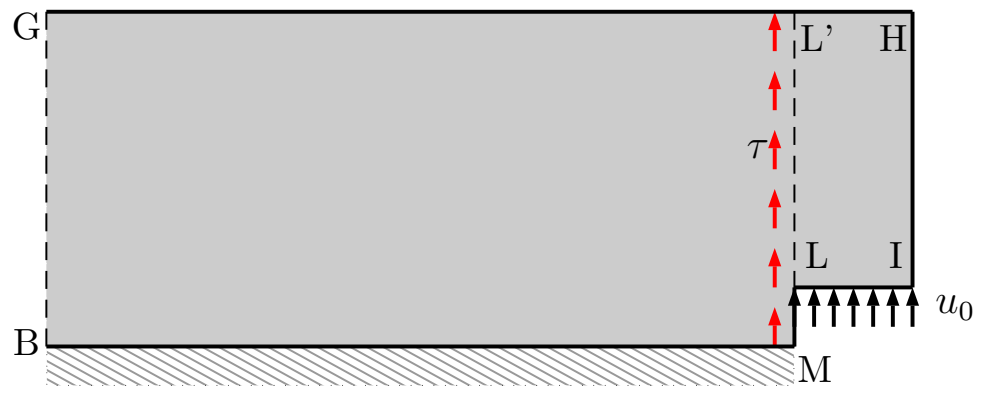

Figure 10: Resultant force scheme for initial loading due to the imposed displacement $u_{0}$.

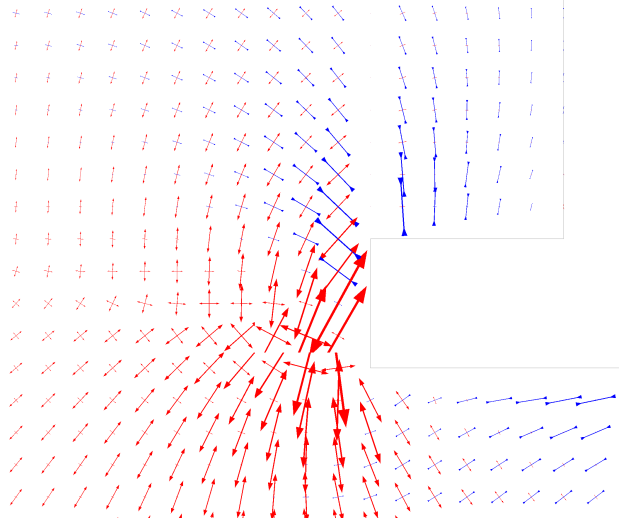

(a)

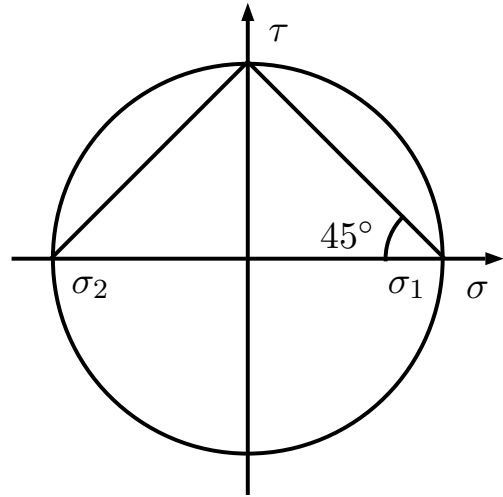

(b)

Figure 11: Stress vectors in the elastic case (left) and Mohr's circle at point M (right). The pure shear condition creates a stress state characterized by principal directions inclined at $45^{\circ}$ with the horizontal.

the crack follows the direction orthogonal to the maximum tensile stress. Therefore, a crack initiates in $\mathrm{M}$ and progresses at $45^{\circ}$. The force scheme changes as depicted in Figure 12. From the global equilibrium of the block M'B'GL', it is possible then to infer that a new shear stress $\tau^{\prime}$, in a similar fashion as before. The crack will consequently follow a 45 degree line.

This crack pattern is typical of the symmetric solution in the pullout test. In fact, when the imposed displacement is applied in such a way that horizontal forces are very small or lateral reactions are equilibrated, the stress state transferred from the steel head is close to pure shear. Whether the concrete panel is effectively constrained by a steel bar at the line GB, located far from the applied shear, the same solution pattern is expected, with a crack growing diagonally at approximately $45^{\circ}$. Considering Poisson's effect, normal and shear stress-transfer across the fissured crack makes the problem more complex and obtaining an analytical solution is unfeasible. However, the expected solution does not depart significantly from the upward crack at approximately $45^{\circ}$.

Figure 13 and 14 show contours for the equivalent plastic strain and displacements obtained with mixed (top row) and standard (bottom row) finite elements in two situations: with (left) and without 


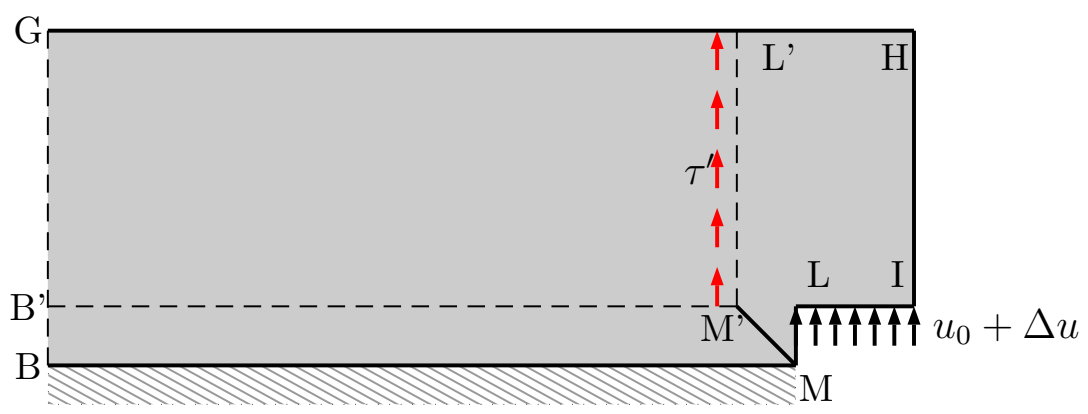

Figure 12: Forces scheme for the initiated crack

(right) steel rod bracing.

All the solutions attempt to reproduce a crack that initiates at point $\mathrm{M}$ and progresses upward (Figure 13), inducing the rotation of the detaching part around the tip of the crack (Figure 14). However, the results produced by the mixed and the standard formulations are very different.

On the one hand, the mixed finite elements produce results which are: (a) consistent with those expected from the (simplified) analytical solution; (b) correctly insensitive to the change in the bracing system; (c) correctly insensitive to the mesh alignment.

On the other hand, the standard formulation produces unreliable results that are: (a) not consistent with the expected crack at approximately $45^{\circ}$; (b) spuriously sensitive to the stress state induced by the bracing; (c) spuriously sensitive to the alignment of the mesh.

Figure 15 shows the force $F$ versus the vertical displacement $u$ for the four cases. The mixed formulation produces very consistent results, with very limited effect of the bracing system in the formation of the crack. The standard formulation overestimates the peak load and the energy dissipated in the formation of the crack.

It has to be noted that the reaction force cannot vanish because the compression stresses are not affected by the adopted Rankine criterion. This means that the crack progresses towards the upper surface but it can not reach it. 


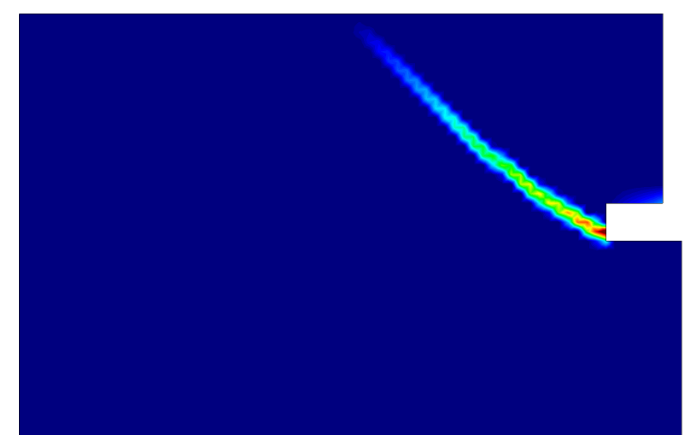

(a) $\varepsilon / u$ FEM - Bracing

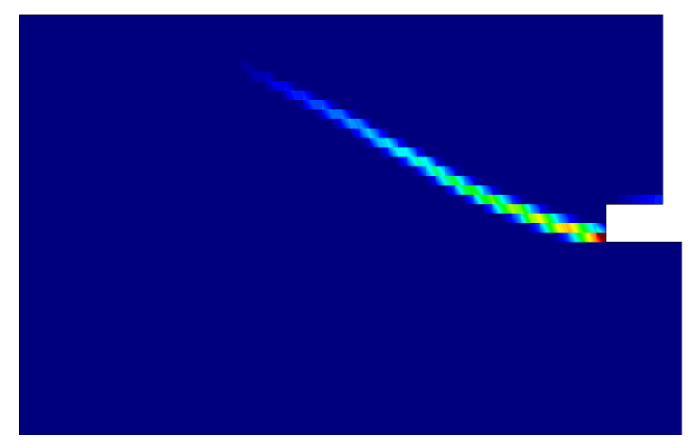

(c) Std FEM - Bracing

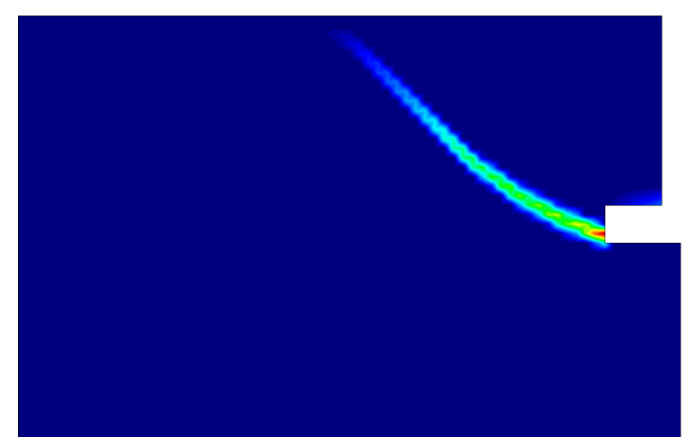

(b) $\varepsilon / u$ FEM - No bracing

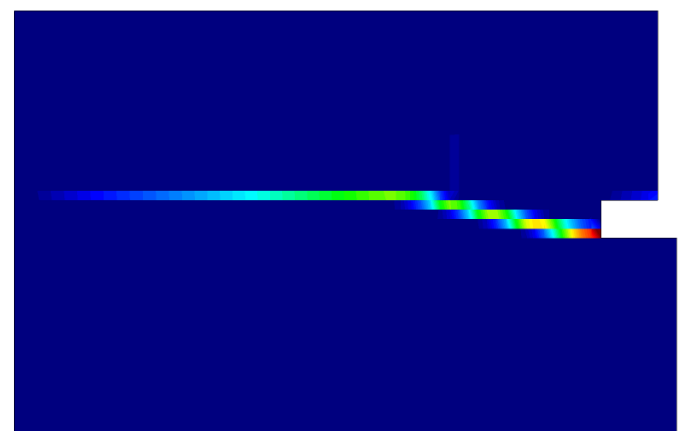

(d) Std FEM - No Bracing

Figure 13: Equivalent plastic strain contours with mixed (top row) and the standard (bottom row) finite element formulations in the pure shear case.

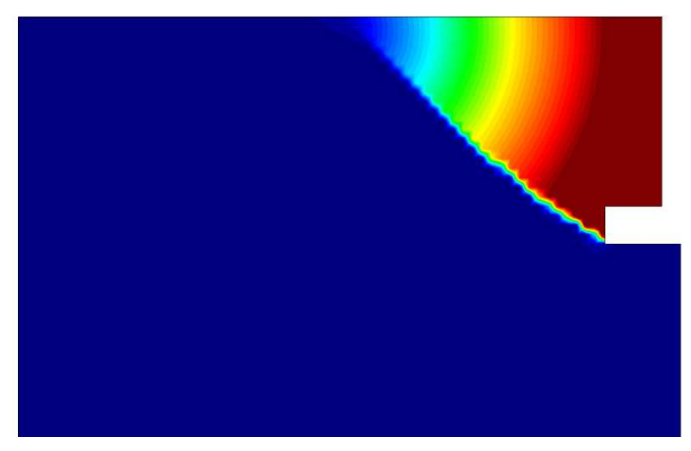

(a) $\varepsilon / u$ FEM - Bracing

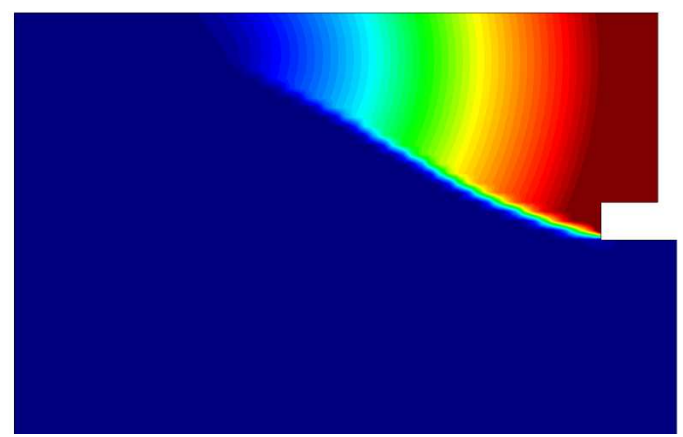

(c) Std FEM - Bracing

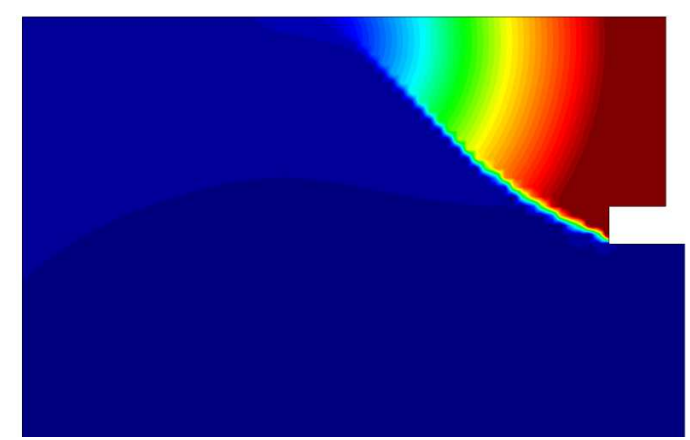

(b) $\varepsilon / u$ FEM - No bracing

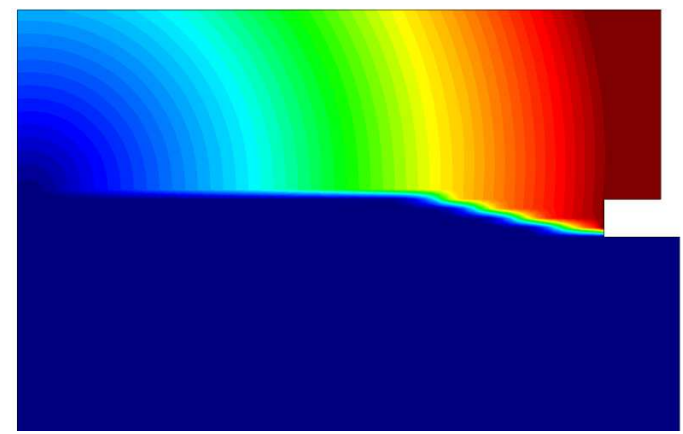

(d) Std FEM - No bracing

Figure 14: Displacements contours with mixed (top row) and the standard (bottom row) finite element formulations in the pure shear case. 


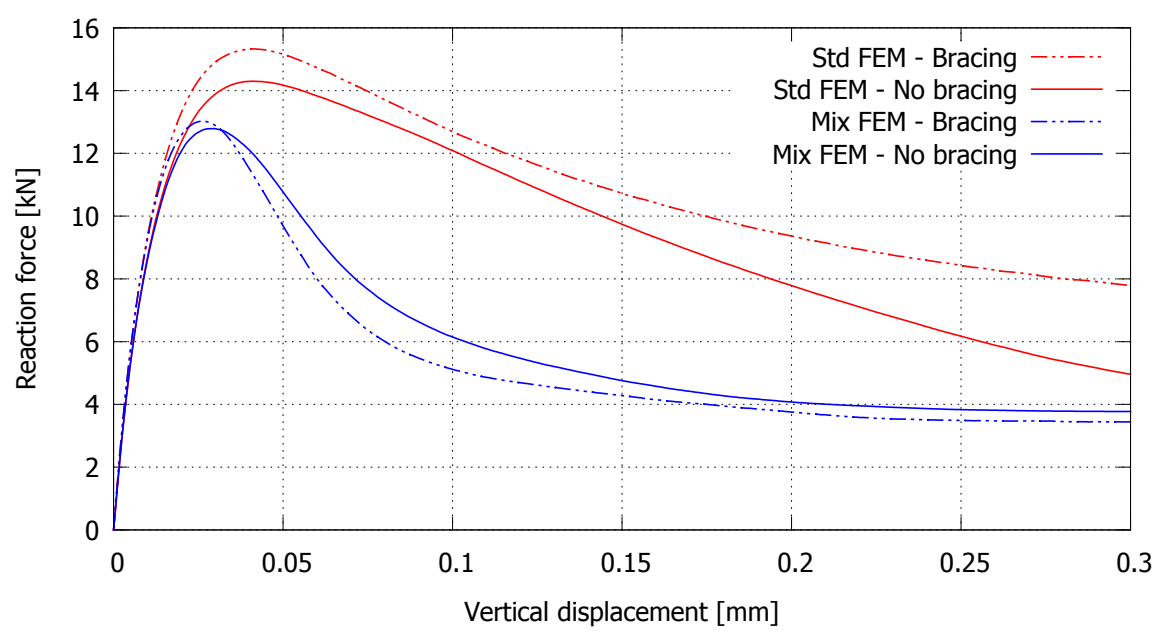

Figure 15: Force versus vertical displacement curves for the pure shear case.

\subsubsection{Simulation of the experimental results}

In this section, the horizontal displacement of the embedded steel head is restrained, to simulate the high friction between the anchored head and the surrounding concrete. Contrariwise to the cases considered in the previous section, in this situation the steel bracing counterbalances the effect of the applied pullout vertical force, as it supplies an opposed downward concentrated force. This force is proportional to the stiffness of the metal rods and, therefore, the resulting fracture pattern is effectively affected by the rigidity of the bracing system.

Figure 16 and 17 show contours for the equivalent plastic strain and displacements obtained with mixed (top row) and standard (bottom row) finite elements in the two cases given by stiff or loose support frames. For the case for a stiff support, the resulting fracture path is curved, from the base of the bolt to the bearing structure (Figure 16(a) and Figure 16(c)). Both mixed and standard finite elements are able to capture the displacement of the mass of concrete under bending (Figure 17(a) and Figure 17(c)). Even if the two solutions appear similar, it is important to notice two details. First, a clear mesh dependency is visible in the standard solution (Figure 16(c)) while this is not the case in the mixed formulation, with a defined inclination at the crack tip (Figure 16(a)). Second, the proposed formulation produces a crack that turns effectively in the direction of the support, while the curvature given by the standard one is reduced, affected by mesh dependence.

In the case of low rigidity, the solution is qualitatively similar in terms of direction of the crack between the two methods, but it is possible to observe a bifurcation in the crack path for the mixed formulation (Figure 16(b)) which is not captured for the irreducible one (Figure 16(d)). The rotation of the detaching part, due primarily to bending effects, is evident also in this case (Figure 17(b) and Figure 17(d)).

In Figures 18, 19 and 20, the numerical solutions of the more reliable mixed $\varepsilon / u$ finite elements are compared with the experimental results. The agreement between numerical solutions and the experimental results is remarkably good. This is achieved even if the adopted constitutive model (Rankine plasticity under tension) does not include phenomena like compressive crushing or friction and dilatation due to grain effects.

In Figure 18, the symmetric pattern (Figure 5) is compared against the solution found in Figure 13(a). With a distant positioning of the bracing and no horizontal restraint, the result is approximately a $45^{\circ}$ inclined crack. Note that a shallower embedding of the flange favours less horizontal constraint, being smaller the vertical reaction (and consequently the frictional forces) of the affected concrete.

In Figure 19, the crack formed with the effective restraint of the steel bracing and zero horizontal sliding of the bolt is presented. The numerical simulation, shown in Figure 19(a), considers a homogeneous material whereas the intrinsic heterogeneity of concrete, with an internal distribution of aggregates, affects the direction of the crack observed in Figure 19(b). 


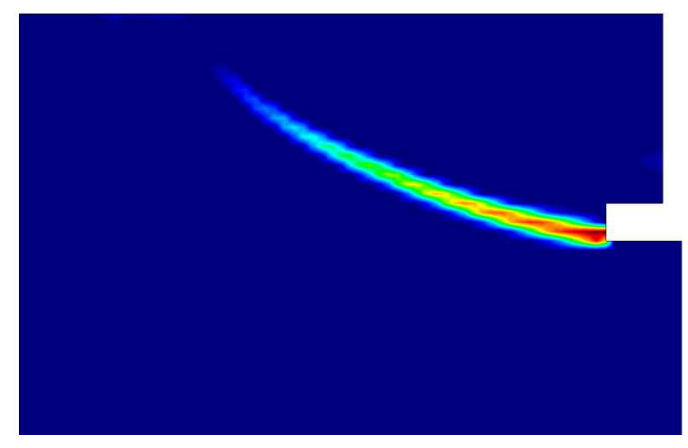

(a) $\varepsilon / u$ FEM - High $E_{b}$

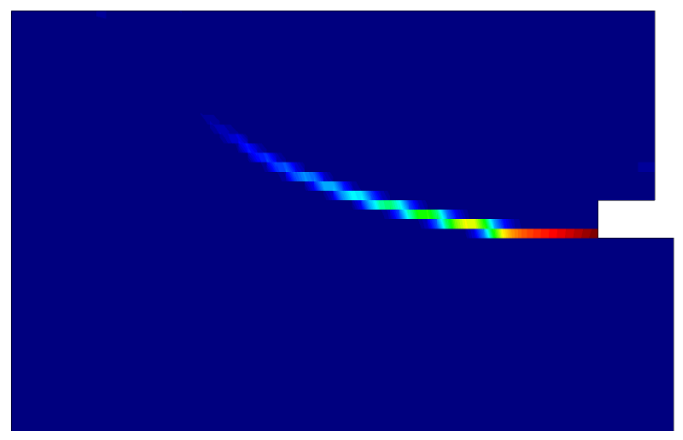

(c) Std FEM - High $E_{b}$

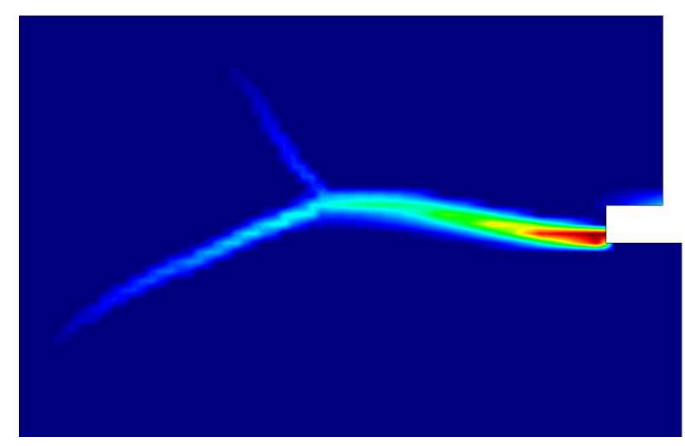

(b) $\varepsilon / u$ FEM - Low $E_{b}$

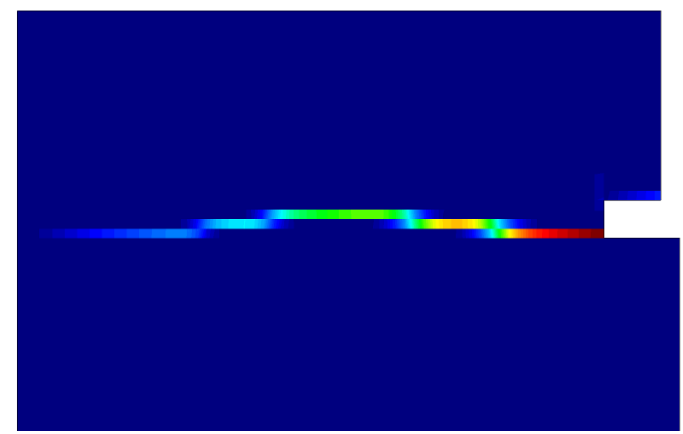

(d) Std FEM - Low $E_{b}$

Figure 16: Equivalent plastic strain contours with mixed (top row) and the standard (bottom row) finite element formulations in the experimental setup case.

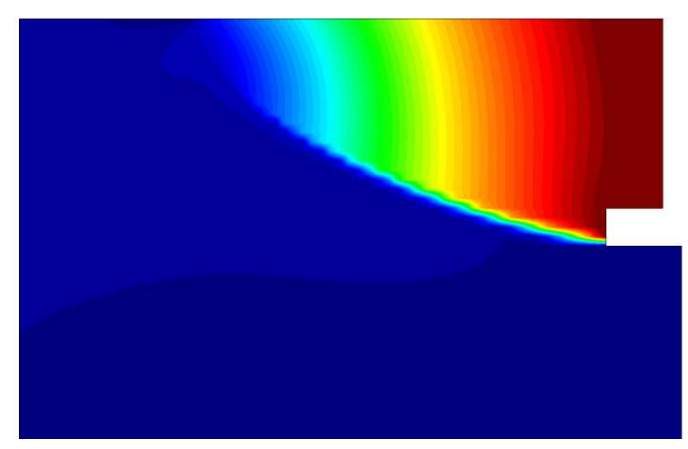

(a) $\varepsilon / u$ FEM - High $E_{b}$

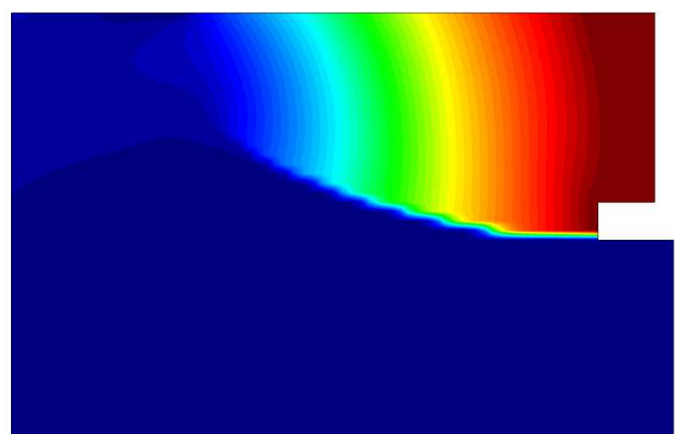

(c) Std FEM - High $E_{b}$

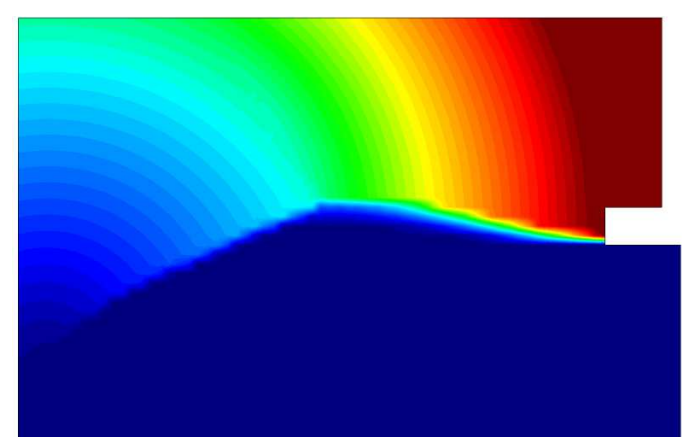

(b) $\varepsilon / u$ FEM - Low $E_{b}$

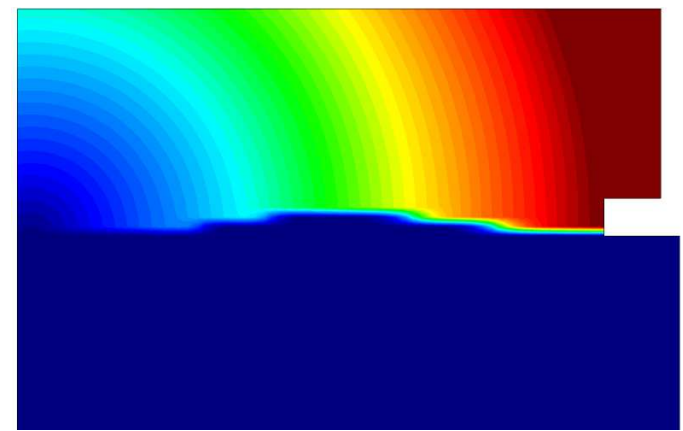

(d) Std FEM - Low $E_{b}$

Figure 17: Displacements contours with mixed (top row) and the standard (bottom row) finite element formulations in the experimental setup case. 
In Figure 20, the bifurcation pattern is shown. This case is due to the low stiffness of the bracing, which implies an initial almost horizontal initiation of the crack, followed by a bifurcation. The longer the horizontal crack path, the bigger the effect of bending is; the slight curvature before the bifurcation point is noticeable. After the bifurcation, the upper part of the crack propagates almost straight to the support bracing. The lower fracture is longer than the upper one in both of the pictures. The agreement between the two results is remarkable.

In Figure 21, the vertical reaction is plotted against the imposed displacement. Two different cases are shown: the first one, corresponding to an effective support action, gives an increasing reaction as the experiment progresses; the second one, corresponding to the bifurcation case, with less stiff bracing, shows a softening behavior. Comparing those results with the ones shown in Figure 15, it is possible to asses the influence of friction and bracing system with respect to the pure shear virtual test. In addition, the peaks load and the dissipated energy fall within the experimental ranges discussed in Dejori [18].

Thanks to the enhanced accuracy provided by the mixed formulation, it is still possible to discuss particular details of the pullout tests, without the need of referring to specific experimental results. For example, the starting point of the crack is determined by the effective contact between the steel flange and the concrete. With reference to Figure 9, in the case of pure vertical motion (i.e. line LI has an imposed displacement, but not LM), the crack starts from point L. Instead, if a slight lateral motion of the head presses on line LM, so that friction makes steel and concrete adherent, then the crack will start from point M. This small horizontal sliding has found to occur frequently for the asymmetric configuration of cracks, where the onset of a first diagonal crack on one side provokes a rotation of the other side. This effect is more visible for larger depth of embedding.

Another key aspect is the sequence of cracking; it is statistically impossible to reach failure at the same time on both sides of the panel due to the natural imperfections in the positioning of the force and in the composition of the concrete. Perturbations largely affect brittle crack propagation and this is reflected by the greater percentage of asymmetric results obtained in the experiments. Nevertheless, a sound hypothesis on the probable sequence of failure can be outlined thanks to the previous examples. The initial cracking is given by a tensile failure as in Figure 18, for the symmetric case, or as in Figure 19, for the asymmetric one. Then, in the latter case, after the first fracture develops, the loading acts unevenly on the two opposite side of the panel and the consequent bifurcating cracks grow in the direction of the steel bracing due to bending-like effects.

\subsection{D Pullout Test}

3D pullout tests have been considered in many publications treating numerical methods for crack propagation. Gasser and Holzapfel [20] analyzed it with the partition of the unity method. Later, Areias and Belytschko [21] and Duan et al. [58] studied it using extended finite elements. Recently, Kaczmarczyk et al. [59] solved the problem with a configurational mechanics method, consistent with Griffith's theory.

The problem, shown in Figure 22, is axis-symmetric, so that only a quarter is depicted. A steel bolt with circular head is placed in a cylindrical concrete anchorage and imposes a vertical displacement to the block. To avoid rigid body motion, a metal hoop is placed on top, reacting vertically. In the same picture, the mechanical properties are reported as taken from Areias and Belytschko [21]. Vertical displacement is imposed, whereas no horizontal displacement is allowed at the contact between the bolt and the concrete.

Here, the problem is studied with three different mesh sizes and comparing the results obtained with mixed $\varepsilon / u$ and standard formulations. The elements used for the discretization are triangular prisms, so that a semi-structured mesh is easily built. Figure 23 shows the top face of the quarter cylinder composed of triangles, supported by a structured vertical square grid. Table 1 shows the average size of the triangles, the number of nodes and elements in three meshes (coarse, medium and fine) so that the convergence of the solution with respect to the mesh sizes can be assessed.

In Figure 24 it is possible to see the computed deformed shape along the resulting conical crack surface in the case of the mixed finite element with fine meshing. The fields of equivalent plastic strain 


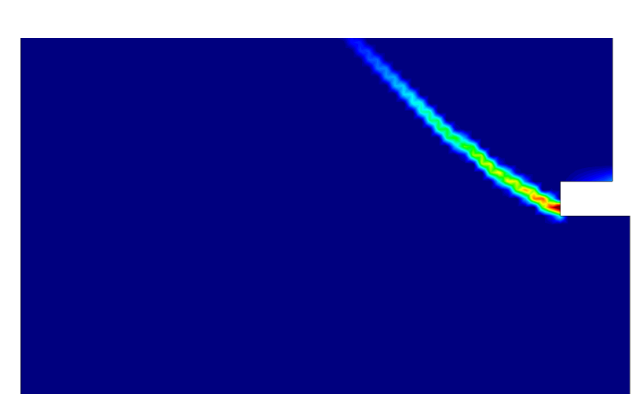

(a)

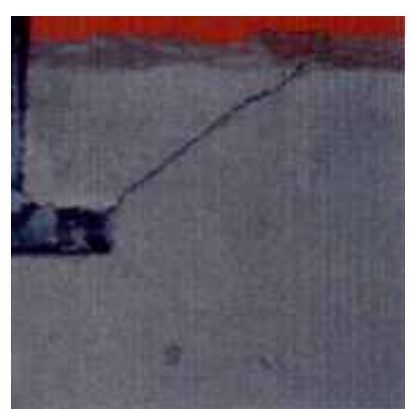

(b)

Figure 18: Comparing the symmetric pattern in the case of free horizontal sliding of the steel flange.

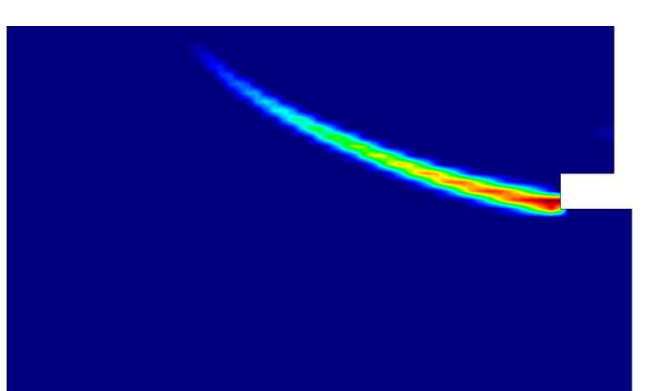

(a)

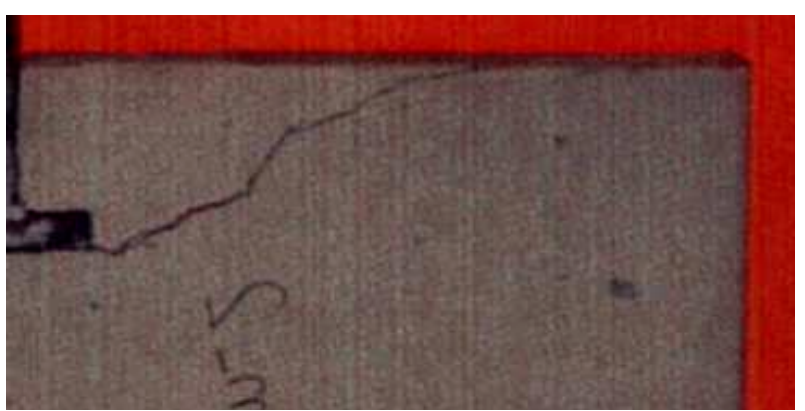

(b)

Figure 19: Comparing the symmetric pattern for the case of effective vertical constraint of steel bracing and constrained horizontal motion of the steel flange.

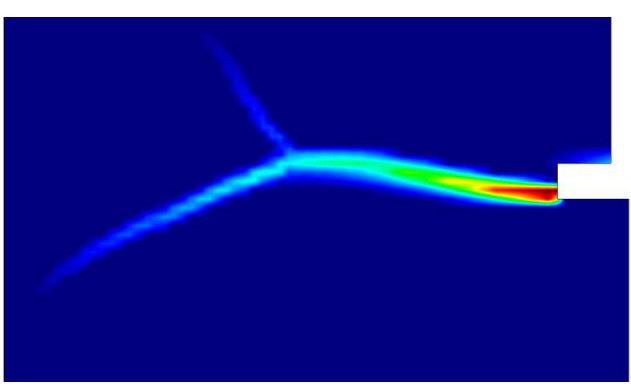

(a)

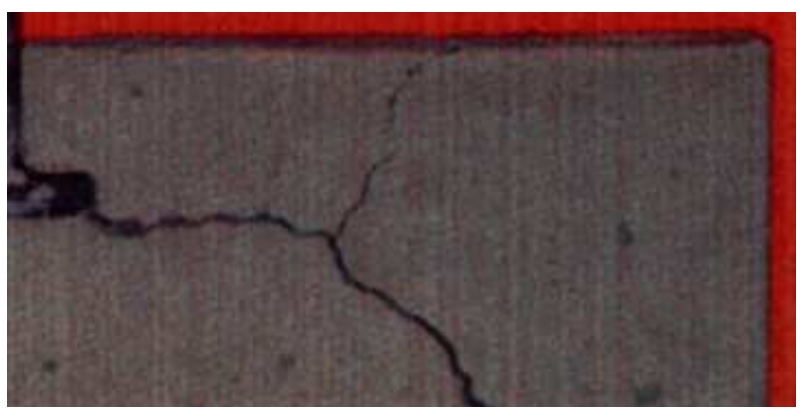

(b)

Figure 20: Comparing the bifurcation pattern in the asymmetric case.

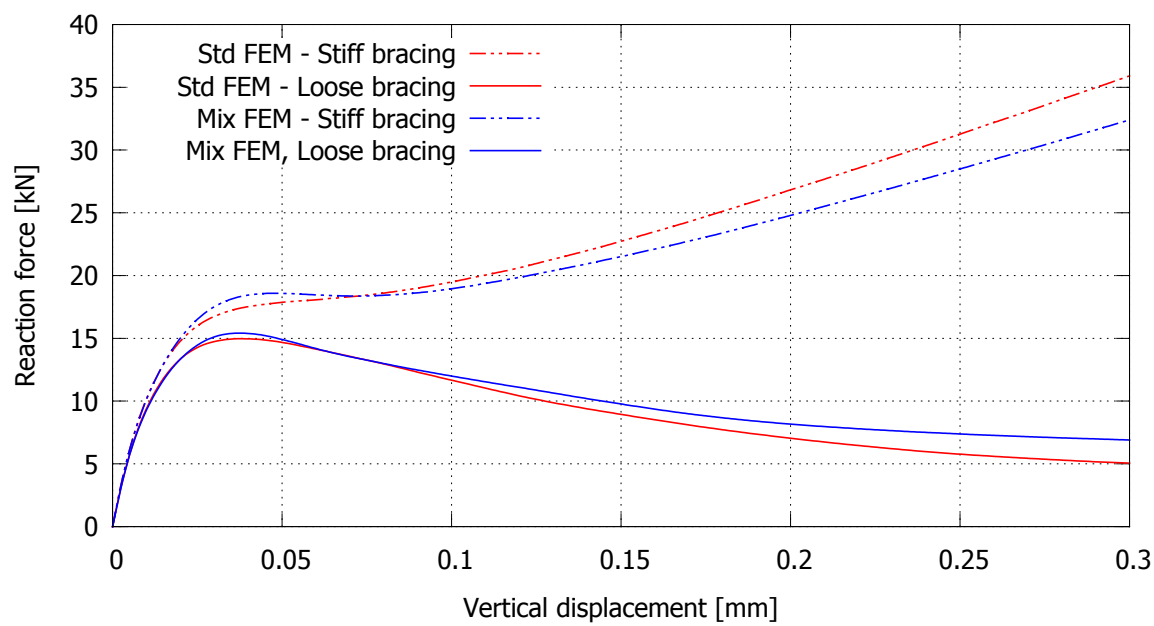

Figure 21: Force versus vertical displacement curves for the experimental case. 


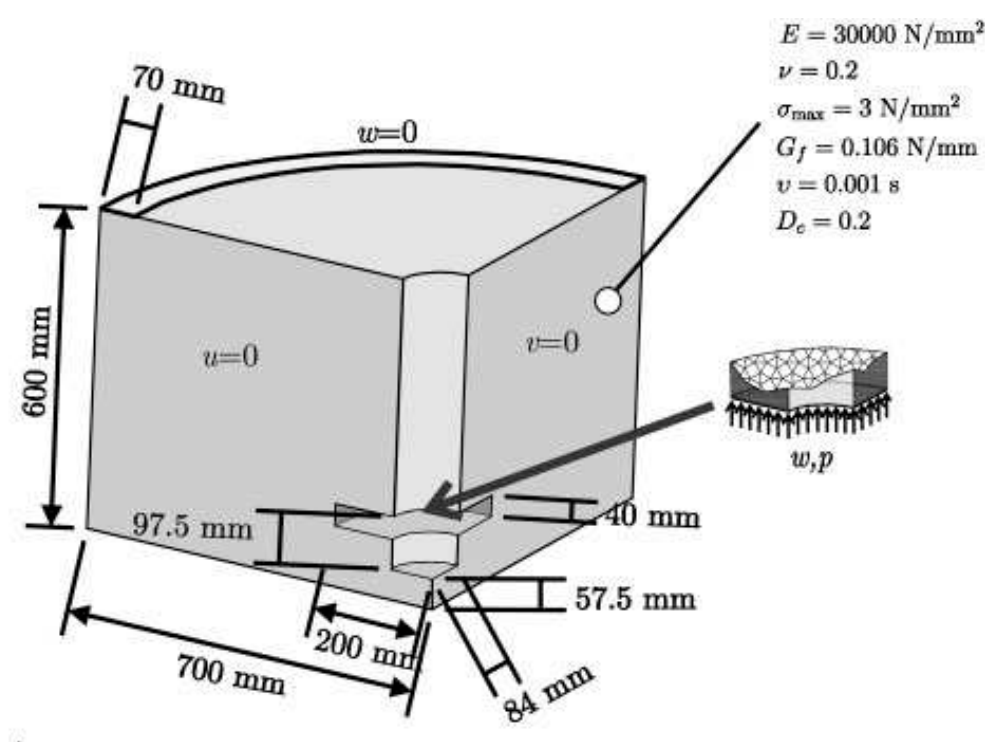

Figure 22: Geometry of the problem and mechanical properties of the concrete (from Areias and Belytschko [21])

\begin{tabular}{|c|c|c|c|}
\hline Mesh & $\mathrm{h}$ & Nodes & Elements \\
\hline Coarse & $40 \mathrm{~mm}$ & 4664 & 7742 \\
\hline Medium & $30 \mathrm{~mm}$ & 11555 & 20166 \\
\hline Fine & $25 \mathrm{~mm}$ & 20672 & 36985 \\
\hline
\end{tabular}

Table 1: Characteristic mesh sizes, number of nodes and number of elements for the 3D pullout test numerical simulation

for each mesh size are presented in Figure 25. As for the 2D case, the robustness provided by the two methods is very different.

The mixed method yields very consistent results throughout the levels of refinement. For the three meshes, a conical crack starts at the bolt head and progresses towards the steel bracing. The inclination of this crack is defined by the physical geometry of the problem. A secondary, more diffuse, vertical crack appears at the top surface. The standard method produces results which are clearly affected by the bias of the discretization adopted. This is evident for the primary and, even worse, for the secondary cracks.

Figure 26 shows the vertical reaction on the bolt with respect to the imposed displacement. The curves from the three different meshes from both methods are presented. It confirms that:

(a) the mixed formulation provides convergent results; even relatively coarse meshes can produce fairly accurate results, in good agreement with reported numerical simulations;

(b) the standard formulation is severely affected by the discretization adopted, both qualitatively and quantitatively; the corresponding curves show significant over-dissipation.

Finally, it is interesting to observe the similarity in the plots of the reaction forces, between the 2D test (in Figure 21) and 3D test (Figure 26).

\section{Conclusion}

High-fidelity simulations of 2D and 3D pullout tests on concrete are presented. An elasto-plastic model with Rankine criterion is adopted as constitutive law; details on the formulation and regularization of edge and apex are given. 


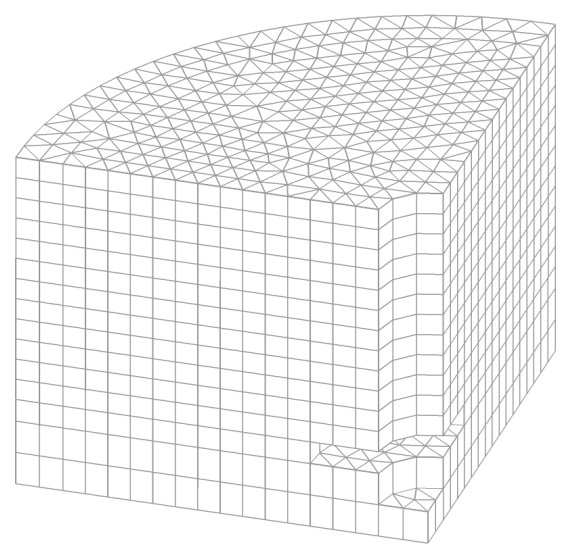

(a)

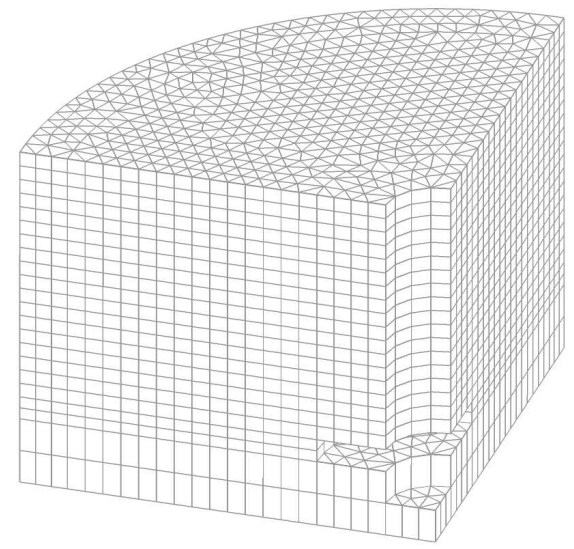

(b)

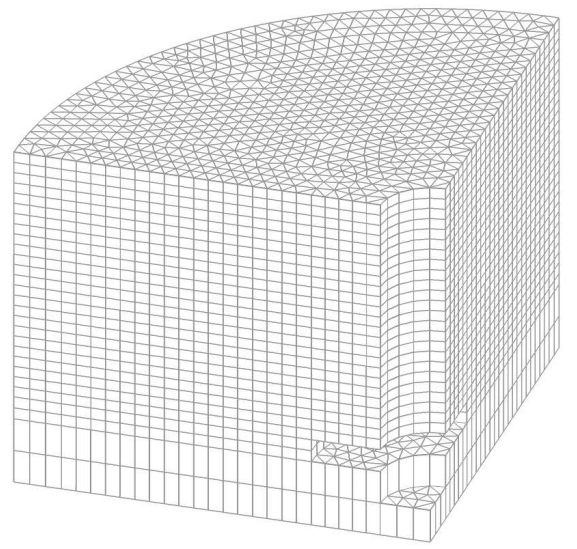

(c)

Figure 23: Discretization meshes for the 3D pullout problem: from left to right, coarse mesh (a), medium mesh (b) and fine mesh (c).

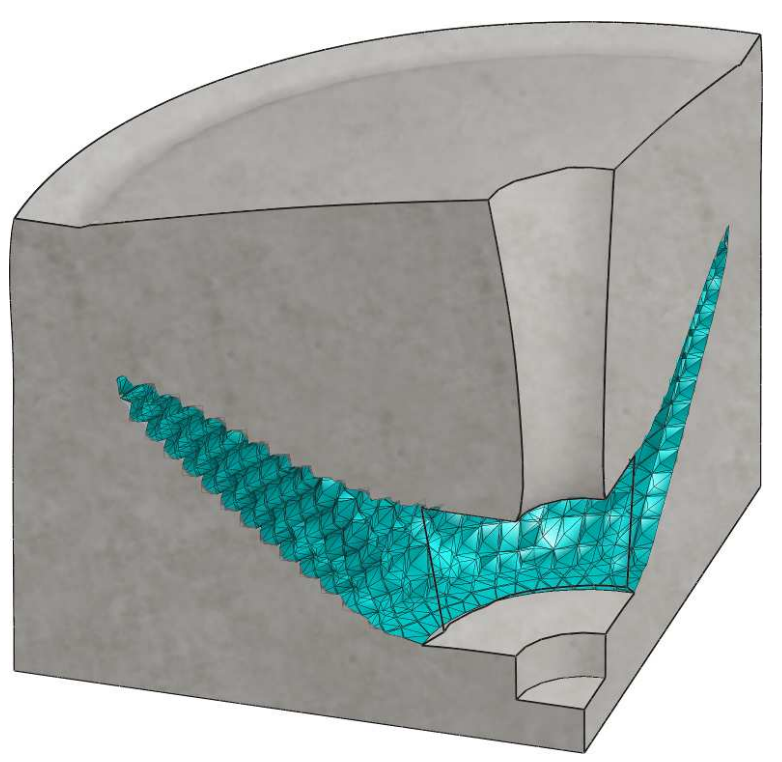

(a)

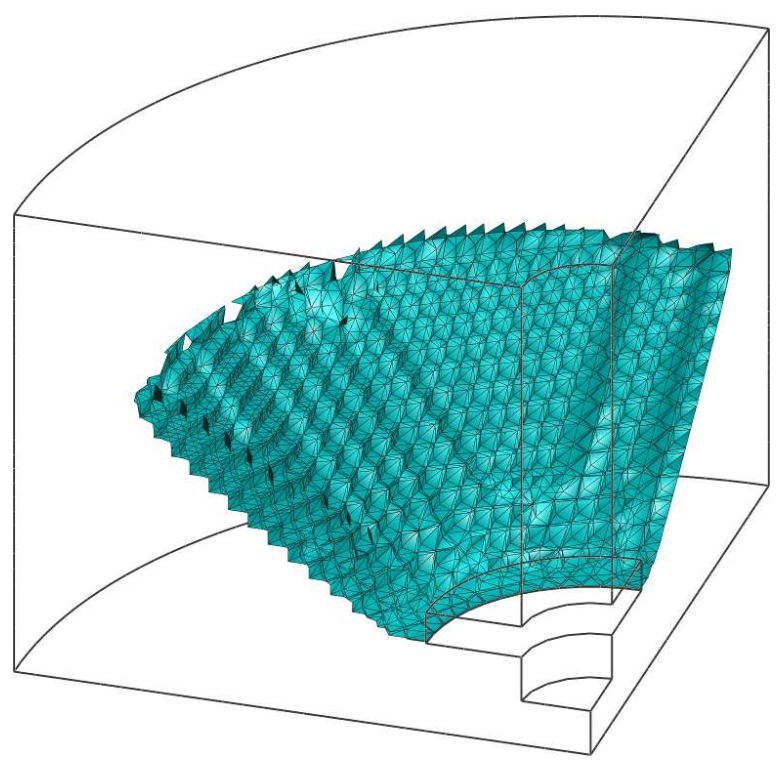

(b)

Figure 24: Deformed concrete block with the crack opening (left) and crack conical surface (right) resulting from the mixed formulation analysis with fine mesh. 


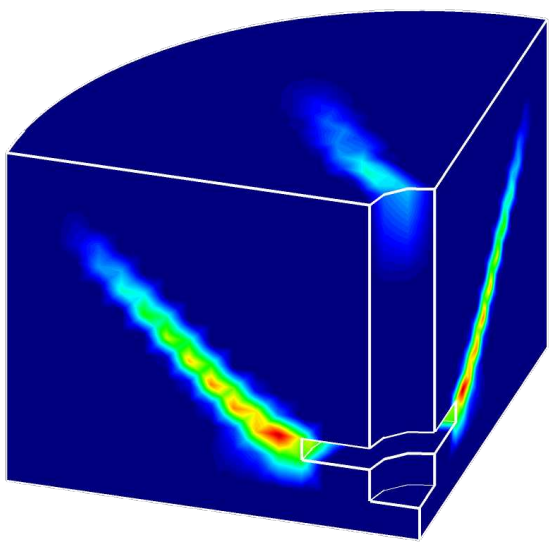

(a)

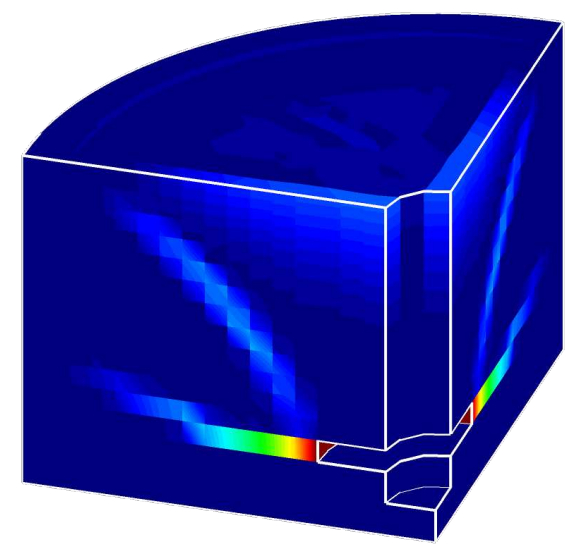

(d)

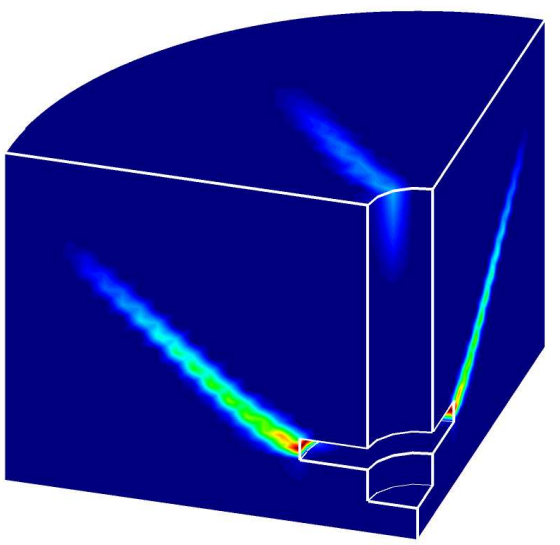

(b)

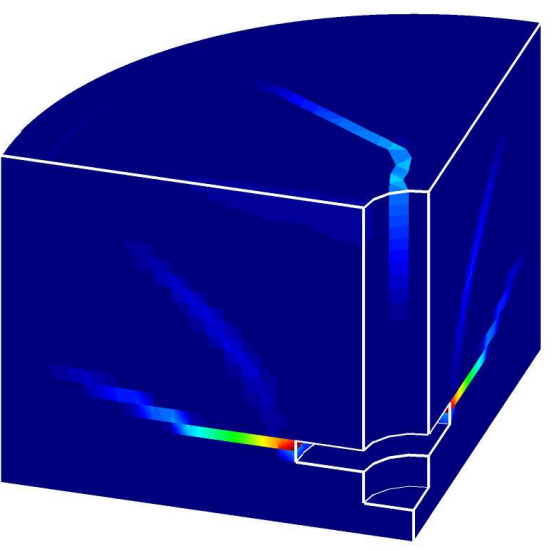

(e)

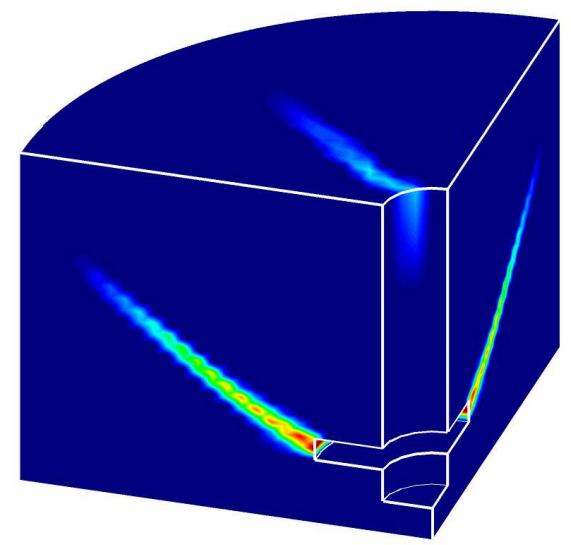

(c)

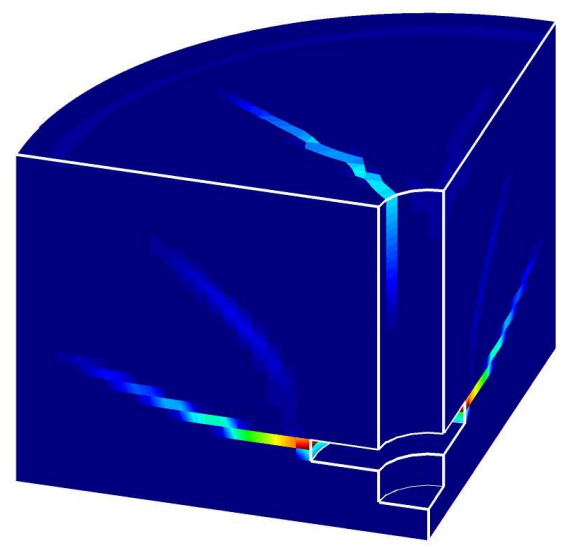

(f)

Figure 25: Equivalent plastic strains for the mixed (top) and the standard formulations (bottom).

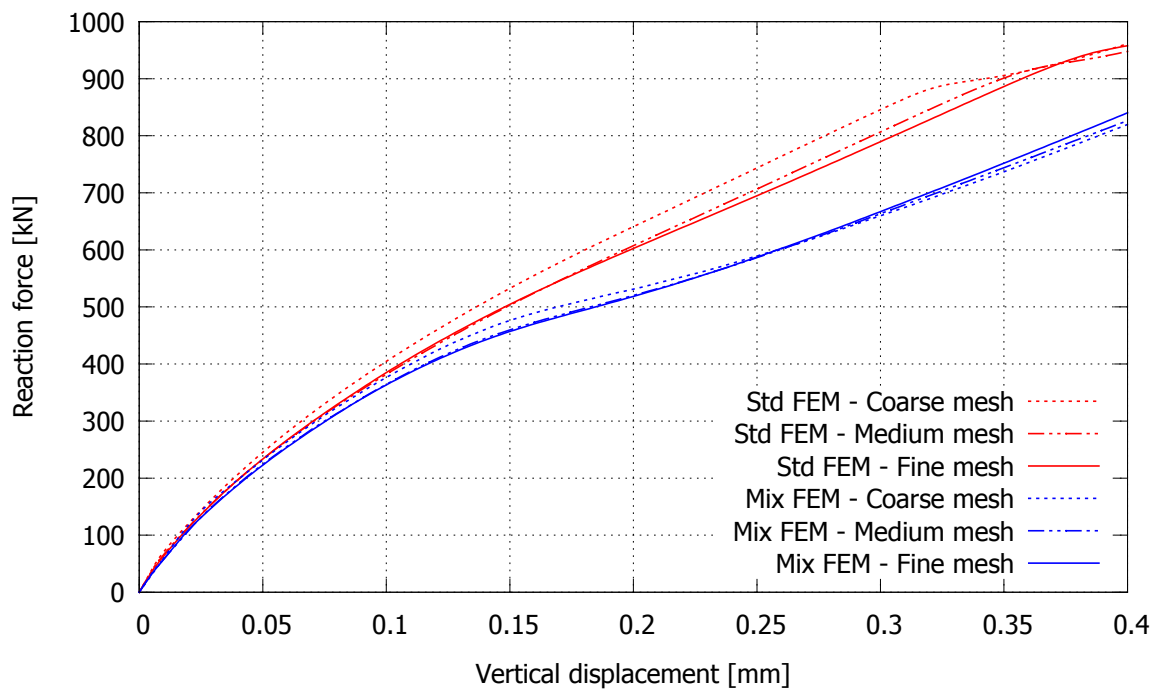

Figure 26: Reaction versus displacement curve for the 3D pullout test. 
A novel mixed strain/displacement formulation is used; the necessary stabilization procedure to ensure stability with equal order interpolation spaces is described and implementation issues are covered. The enhanced convergence and accuracy properties of the mixed formulation are demonstrated through the numerical simulation of plane and axis-symmetrical pullout tests. The mixed method yields accurate results, on the one side, showing sensitivity to the physical parameters of the tests, and, on the other side, without spurious sensitivity to the mesh adopted.

In all cases, the expected cracks are predicted as plastic-strain localization bands, without the need of using ad-hoc auxiliar tracking techniques. Crack bifurcation, in accordance with the experimental results, can also be predicted; this is an impossible feat for the local or global tracking algorithm. In addition, the mixed formulation demonstrates ability to capture the correct failure mechanism and the corresponding peak and post-peak behavior with relative coarse meshes. This is an important consideration on the cost-effectiveness of the formulation.

\section{Acknowledgments}

Financial support from the EC $7^{\text {th }}$ Framework Programme under the MuMoLaDe project - Multiscale Modelling of Landslides and Debris Flows - within the framework of Marie Curie ITN (Initial Training Networks) and the Spanish Ministry of Economy and Competitivity under the EACY project

- Enhanced accuracy computational and experimental framework for strain localization and failure mechanisms- within the "Excellency" Program for Knowledge Generation is gratefully acknowledged. 


\section{References}

[1] B. Winkler, G. Hofstetter, and G. Niederwanger. Experimental verification of a constitutive model for concrete cracking. Proceedings of the Institution of Mechanical Engineers, Part L: Journal of Material Design and Applications, 215:75-86, 2001.

[2] B. G. Trunk. Einfluß der Bauteilgröße auf die Bruchenergie von Beton. Ph.D. thesis, ETH Eidgenössischen Technischen Hochschule Zürich, 1999.

[3] G. V. Guinea, J. Planas, and M. Elices. Measurement of the fracture energy using three-point bend tests: Part 1 - Influence of experimental procedures. Materials and Structures, 25(4):212218, 1992 .

[4] J. C. Gálvez, M. Elices, G. V. Guinea, and J. Planas. Mixed mode fracture of concrete under proportional and nonproportional loading. International Journal of Fracture, 94(3):267-284, 1998.

[5] M. B. Nooru-Mohamed. Mixed-mode fracture of concrete: an experimental approach. Ph.D. thesis, TU Delft, Delft University of Technology, 1992.

[6] E. Ballatore, A. Carpinteri, G. Ferrara, and G. Melchiorri. Mixed mode fracture energy of concrete. Engineering Fracture Mechanics, 35(13):145 - 157, 1990.

[7] R. Berenbaum and I. Brodie. Measurement of the tensile strength of brittle materials. British Journal of Applied Physics, 10(6):281, 1959.

[8] D. A. Abrams. Tests of bond between concrete and steel. Number 71. University of Illinois Bulletin edition, 1913.

[9] W. A. Slater, F. E. Richart, and G. G. Scofield. Tests of bond resistance between concrete and steel. Number 173. Department of Commerce, Technologic papers of the Bureau of Standards edition, 1920.

[10] L. Elfgren, C. E. Broms, H. Johansson, and A. Rehnstrom. Anchor Bolts in Reinforced Concrete Foundations: Short Time Tests. Luleå University of Technology, 1980.

[11] W. H. Peier. Model for pull-out strength of anchors in concrete. Journal of Structural Engineering, 109(5):1155-1173, 1983.

[12] R. Eligehausen and G. Sawade. A fracture mechanics based description of the pull-out behavior of headed studs embedded in concrete. Universitt Stuttgart, 1989.

[13] Z. P. Bažant, J. Ožbolt, and R. Eligehausen. Fracture size effect: review of evidence for concrete structures. Journal of structural engineering, 120(8):2377-2398, 1994.

[14] J. Ožbolt, R. Eligehausen, and H.W. Reinhardt. Size effect on the concrete cone pull-out load. International Journal of Fracture, 95(1):391-404, 1999.

[15] B. L. Karihaloo. Pull-out of axisymmetric headed anchors. Materials and Structures, 29(3): 152-157, 1996.

[16] M. Baena, L. Torres, A. Turon, and C. Barris. Experimental study of bond behaviour between concrete and FRP bars using a pull-out test. Composites Part B: Engineering, 40(8):784-797, 2009 .

[17] T. Lecompte, A. Perrot, A. Subrianto, A. Le Duigou, and G. Ausias. A novel pull-out device used to study the influence of pressure during processing of cement-based material reinforced with coir. Construction and Building Materials, 78:224-233, 2015. 
[18] M. Dejori. Experimentelle und numerische Traglastanalysen von axial beanspruchten Kopfbolzen beim Versagensmechanismus Betonausbruch. Diplomarbeit (Master thesis), University of Innsbruck, 2006.

[19] Y. Thenier and G. Hofstetter. Numerical prediction of crack propagation and crack widths in concrete structure. Engineering Structures, 31:1832-1840, 2009.

[20] T. C. Gasser and G. A. Holzapfel. Modeling 3D crack propagation in unreinforced concrete using PUFEM. Computer methods in applied mechanics and engineering, 194:2859-2896, 2005.

[21] P. M. A. Areias and T. Belytschko. Analysis of three-dimensional crack initiation and propagation using the extended finite element method. International journal for numerical methods in engineering, 63:760-788, 2005.

[22] M. Cervera, M. Chiumenti, L. Benedetti, and R. Codina. Mixed stabilized finite element methods in nonlinear solid mechanics. Part III: Compressible and Incompressible Plasticity. Computer Methods in Applied Mechanics and Engineering, 285:752-775, 2015.

[23] L. Benedetti, M. Cervera, and M. Chiumenti. Stress-accurate Mixed FEM for soil failure under shallow foundations involving strain localization in plasticity. Computers and Geotechnics, 64: $32-47,2015$.

[24] Y. R. Rashid. Ultimate strength analysis of prestressed concrete pressure vessels. Nuclear Engineering and Design, 7(4):334-344, 1968.

[25] M. Cervera and M. Chiumenti. Size effect and localization in J2 plasticity. International Journal of Solids and Structures, 46(17):3301-3312, August 2009.

[26] M. Cervera, M. Chiumenti, and R. Codina. Mixed stabilized finite element methods in nonlinear solid mechanics. Part II: Strain localization. Computer Methods in Applied Mechanics and Engineering, 199(37-40):2571-2589, August 2010.

[27] M. Cervera, M. Chiumenti, and R. Codina. Mesh objective modeling of cracks using continuous linear strain and displacement interpolations. International Journal for Numerical Methods in Engineering, (87):962-987, 2011.

[28] R. Faria, J. Oliver, and M. Cervera. A strain-based plastic viscous damage model for massive concrete structures. International Journal of Solids and Structures, 35(14):1533-1558, 1998.

[29] C. Feist and G. Hofstetter. An embedded strong discontinuity model for cracking of plain concrete. Computer Methods in Applied Mechanics and Engineering, 195(52):7115 - 7138, 2006.

[30] P. H. Feenstra and R. de Borst. A plasticity model and algorithm for mode-I cracking in concrete. International Journal for Numerical Methods in Engineering, 38:2509-2529, 1995.

[31] B. Winkler, G. Hofstetter, and H. Lehar. Application of a constitutive model for concrete to the analysis of a precast segmental tunnel lining. International Journal for Numerical and Analytical Methods in Geomechanics, 28:797-819, 2004.

[32] J. C. Simo and T. J. R. Hughes. Computational Inelasticity. Interdisciplinary applied mathematics: Mechanics and materials. Springer New York, 1998.

[33] D. Boffi, F. Brezzi, and M. Fortin. Mixed Finite Element Methods and Applications. Springer, 2013 edition, 72013.

[34] T. J. R. Hughes, G. R. Feijóo, L. Mazzei, and J. B. Quincy. The variational multiscale method - A paradigm for computational mechanics. Computer Methods in Applied Mechanics and Engineering, 7825(98), 1998. 
[35] R. Codina. Stabilization of incompressibility and convection through orthogonal sub-scales in finite element methods. Computer Methods in Applied Mechanics and Engineering, 190:1579$1599,2000$.

[36] S. Badia and R. Codina. Unified stabilized finite element formulations for the Stokes and the Darcy problems. SIAM Journal on Numerical Analysis, 47(3):1971-2000, 2009.

[37] R. Codina. Finite element approximation of the three-field formulation of the Stokes problem using arbitrary interpolations. SIAM Journal on Numerical Analysis, 47(1):699-718, 2009.

[38] M. Cervera, M. Chiumenti, Q. Valverde, and C. Agelet de Saracibar. Mixed linear/linear simplicial elements for incompressible elasticity and plasticity. Computer Methods in Applied Mechanics and Engineering, 192(49-50):5249-5263, 122003.

[39] R. Codina. Analysis of a stabilized finite element approximation of the Oseen equations using orthogonal subscales. Applied Numerical Mathematics, 58(3):264 - 283, 2008.

[40] M. Cervera, M. Chiumenti, and R. Codina. Mixed stabilized finite element methods in nonlinear solid mechanics. Part I: Formulation. Computer Methods in Applied Mechanics and Engineering, 199(37-40):2559-2570, August 2010.

[41] J. W. Ju. Isotropic and anisotropic damage variables in continuum damage mechanics. Journal of Engineering Mechanics, 116(12):2764-2770, 1990.

[42] G. Meschke, R. Lackner, and H. A. Mang. An anisotropic elastoplastic-damage model for plain concrete. International Journal for Numerical Methods in Engineering, 42(4):703-727, 1998.

[43] L. Berto, A. Saetta, R. Scotta, and R. Vitaliani. An orthotropic damage model for masonry structures. International Journal for Numerical Methods in Engineering, 55(2):127-157, 2002.

[44] E. Rizzi and I. Carol. Dual orthotropic damageeffect tensors with complementary structures. International Journal of Engineering Science, 41(1314):1445 - 1495, 2003.

[45] L. Pelà, M. Cervera, and P. Roca. Continuum damage model for orthotropic materials: Application to masonry. Computer Methods in Applied Mechanics and Engineering, 200(9):917-930, 2011.

[46] J. G. Rots, P. Nauta, G. M. A. Kuster, and J. Blaauwendraad. Smeared crack approach and fracture localization in concrete. HERON, 30 (1), 1985, 1985.

[47] J. Oliver. A consistent characteristic length for smeared cracking models. International Journal for Numerical Methods in Engineering, 28(2):461-474, 1989.

[48] M. Cervera, J. Oliver, and R. Faria. Seismic evaluation of concrete dams via continuum damage models. Earthquake engineering \& structural dynamics, 24(9):1225-1245, 1995.

[49] R. Faria, J. Oliver, and M. Cervera. A strain-based plastic viscous-damage model for massive concrete structures. International Journal of Solids and Structures, 35(14):1533-1558, 1998.

[50] R. de Borst. Integration of plasticity equations for singular yield functions. Computers and Structures, 26(5):823 - 829, 1987. ISSN 0045-7949.

[51] H. Matsuoka and T. Nakai. Stress-deformation and strength characteristics of soil under three different principal stresses. Proc. JSCE, 232:57-70, 1974.

[52] J. M. Gesto, A. Gens, and J. Vaunat. Smoothing of yield surface and a reformulation of multisurface plasticity. In E. Oñate, D. R. J. Owen, D. Peric, and B. Suárez, editors, Proceedings of the XI International Conference on Computational Plasticity. Fundamentals and Applications. CIMNE - International Center of Numerical Methods in Engineering, 2011. 
[53] C. Miehe. Numerical computation of algorithmic (consistent) tangent moduli in large-strain computational inelasticity. Computer Methods in Applied Mechanics and Engineering, 134(34): 223-240, 1996.

[54] A. Pérez-Foguet, A. Rodríguez-Ferran, and A. Huerta. Numerical differentiation for local and global tangent operators in computational plasticity. Computer Methods in Applied Mechanics and Engineering, 189(1):277-296, 2000.

[55] T. W. Reps and L. B. Rall. Computational divided differencing and divided-difference arithmetics. Higher-Order and Symbolic Computation, 16(1-2):93-149, 2003. ISSN 1388-3690.

[56] S. Pietruszczak and Z. Mróz. Finite element analysis of deformation of strain-softening materials. International Journal for Numerical Methods in Engineering, 17(3):327-334, 1981.

[57] M. Cervera, C. Agelet de Saracibar, and M. Chiumenti. COMET: Coupled Mechanical and Thermal analysis. Data Input Manual, Version 5.0, Technical Report IT-308. 2002. URL http://www. cimne.com/comet/.

[58] Q. Duan, J.-H. Song, T. Menouillard, and T. Belytschko. Element-local level set method for threedimensional dynamic crack growth. International journal for numerical methdod in engineering, $80: 1520-1543,2009$.

[59] L. Kaczmarczyk, M. M. Nezhad, and C. Pierce. Three dimensional brittle fracture: configurational-force-driven crack propagation. International Journal for Numerical Methods in Engineering, 97:531-550, 2014. 